\title{
Functional renormalization group for quantized anharmonic oscillator
}

\author{
S. Nagy and K. Sailer \\ Department of Theoretical Physics, University of Debrecen, Debrecen, Hungary
}

(Dated: May 23, 2018)

\begin{abstract}
Functional renormalization group methods formulated in the real-time formalism are applied to the $O(N)$ symmetric quantum anharmonic oscillator, considered as a $0+1$ dimensional quantum field-theoric model, in the next-to-leading order of the gradient expansion of the one- and twoparticle irreducible effective action. The infrared scaling laws and the sensitivity-matrix analysis show the existence of only a single, symmetric phase. The field-independent term of the wavefunction renormalization turned out to be negligible, but its field-dependent piece is noticeable. It is shown that the infrared limits of the running couplings depend on the renormalization group scheme used, when the perturbation expansion in the bare quartic coupling is truncated keeping the terms up to the second order.
\end{abstract}

PACS numbers: 11.10.Gh,11.10.Hi,03.65.-w

\section{INTRODUCTION}

Our main goal in this paper is to investigate the role of wave-function renormalization in the quantum properties of the quartic anharmonic oscillator considering it as a 0 (space)+1(time)-dimensional quantum field-theoric model, and applying various internal space renormalization group (RG) schemes to the one- (1PI) and two-particle irreducible (2PI) effective actions in the truncated gradient expansion which goes beyond the local potential approximation (LPA) with the incorporation of wave-function renormalization terms. In that sense it is a continuation of the work presented in [1]. On the one hand, we apply a Callan-Symanzik (CS) type internal space (IS) RG scheme to the 1PI effective action, where an imaginary mass parameter serves as the control parameter of the RG evolution. On the other hand, another IS RG scheme is applied to the 2PI effective action, where the bare quartic coupling $g_{B}$ represents the control parameter. In both cases, the gradient expansion is used keeping the wavefunction renormalization with, and the local potential is truncated beyond its quartic term. The results obtained in the framework of various RG schemes in the second-order perturbation expansion in the coupling $g_{B}$ are also compared.

The quantized anharmonic oscillator is an elementary toy model representing a building block of more sophisticated and/or realistic models finding several physical applications. Let us mention a few of them without pretending to completeness. The investigation of quantum properties of nanomechanical oscillators has became of interest in order to understand the quantum behaviour of nanodevices [2]. The statistical physical properties of chains of anharmonic oscillators, that of a one-dimensional Ginzburg-Landau system with quartic anharmonicities is of great interest in solid state physics. 2- and 3-dimensional aggregates of such chains describe anisotropic anharmonic solids (e.g. ultrathin ferroelectric films). Putting such systems in inhomogeneous external field enables one to manipulate individual monomers, making such films very attractive for molecular electronics [3]. 1-dimensional Ginzburg -Landau systems are very often investigated by means of the transfer matrix method in which the evaluation of the free energy of an $N+1$ dimensional classical system is equivalent to the evaluation of the ground state energy of an $N$-dimensional quantum system, the so-called dual counterpart of the classical system. In general this dual problem is solved to understand its classical counterpart [4], [5]. Another field where the anharmonic oscillator model has got application is the study of the quantum physics of systems showing up chaotic behaviour classically, ie. the study of quantum chaos [6], [7], [8]. Also the possibility of quantum effects induced chaos has recently been debated on the problem of the quantized Duffing oscillator [9].

These widespreading possibilities of its applications explain the efforts to understand the quantum physics of the anharmonic oscillator in all detail and to invent various theoretical tools for its investigation. Already in the pioneering works [10],[11] it has been pointed out that the Rayleigh-Schrödinger perturbation series for the ground state energy of the quartic anharmonic oscillator diverges. Since then much efforts have been made to overcome that problem and determine the eigenvalues and eigenfunctions of the anharmonic oscillator by various methods like the strongcoupling expansion [12], the integration of operator differential equations [13], multi-scale perturbation theory [14], variational methods [15], iterative Bogoliubov transformations [16], the eigenvalue moment method [17], the improved Hill-determinant method [18], the optimized perturbation expansion [19], a particular iterative method based on the generalized Bloch-equation [20], the auxiliary field method combined with loop expansion [21], as well as the quantum computational method [22]. A class of non-perturbative approaches is based on various RG methods [23-25] including functional RG methods [26, 27], [1] which treat the quantum mechanical problem as a $0+1$ dimensional quantum field-theoric one.

The RG method has been basically developed for the purposes of describing phase transitions and critical behaviour 
in statistical physics and very soon extended to investigate quantum field-theoric models. The RG strategy has been aimed to monitor the Green functions of the elementary field variable when the quantum fluctuation modes are turned on gradually [28]. While in the original version of the RG method a finite fraction of modes are turned on in each blocking steps and one generally relies on the perturbation expansion in bookkeeping the change of the dynamics, the functional RG methods [29]-[32] turn on an infinitesimal fraction of modes in the subsequent blocking steps. Such an approach is accompanied with the usage of infinitely many vertices. The manipulation of a high number of couplings is carried out through their generating functional for which one obtains an exact RG equation looking like a one-loop equation. In order to solve that one has to project it to a suitably chosen functional subspace, e.g. by means of the truncated gradient expansion, but that seems to be a physically better motivated approximation scheme than the truncation of the perturbation expansion. The functional RG strategy has been developed for the 1PI effective action [31], [32] with gliding momentum cut-off. According to the original RG idea one organized the quantum fluctuations according to their wavelengths or momentum, ie. according to their properties in the external space. A natural generalization came when such an ordering has achieved according to their internal-space characteristics, the largeness of their amplitudes. That step has been done by developing the functional generalization of the CS equation [33] where the mass parameter is evolved [34] and the modes of the quantum fluctuations are turned on in the order of their increasing amplitudes. The CS RG scheme and the functional RG schemes with gliding momentum cut-off give similar scaling laws in the leading order in the UV scaling regime where the cutoff is the only scale parameter, but approaching the IR scaling regime the cut-off dependence and the mass-dependence may differ due to the occurring of additional physical scales. A further generalization of the CS RG strategy is called IS RG [35], when any of the parameters of the model can be used as control parameter and evolving it gradually one arrives at a functional RG evolution equation for the 1PI effective action. One should mention that all IS RG schemes including the CS RG compare various theories belonging to the gradually altering values of the control parameter, while the RG schemes with gliding momentum cut-off provide true scaling laws for a given theory. So long the suppression term generating the IS RG evolution is quadratic, the evolution equation contains one-loop integrals. But it can be rather complicated when the suppression term is not quadratic in the elementary field variable since the excitations described by the higher-loop integrals appearing in the evolution equation have rather complicated structure in singleparticle terms. That problem can be overcome applying the internal space RG to the generating functional of some composite operator of the elementary field, in which the suppression term is again quadratic. In particular, in the case of a suppression term being quartic in the elementary field variable, one may apply the RG method to the 2PI effective action [35], the generating functional of 2PI 2-particle correlation functions [36, 37]. That method has the advantage that all expressions are formally ultraviolet (UV) finite until the generally UV divergent loop integrals are made explicit making an Ansatz for the parametrization of the one-particle Green function in whose terms those are expressed.

As mentioned above, various functional RG methods have been recently used and proved to be powerful nonperturbative tools in treating the problem of the quantum anharmonic oscillator. In [26] the effective average action RG scheme [31] and in [27] the Wegner-Houghton (WH) RG method [29] have been applied to the one-dimensional quantum oscillator with quartic anharmonicity in order to determine the energy of the ground state and that of the gap between the first excited state and the ground state and both have been found to increase strictly monotonically with increasing bare quartic coupling $g_{B}$. It has also been thoroughly discussed that even for a symmetric double-well bare potential there exists a unique ground state, so that the quantum anharmonic oscillator exhibits only a single phase. In the WH RG approach one integrates out the high-frequency modes of quantum fluctuations in infinitesimal steps above the gliding sharp momentum cut-off $k$. The method has the disadvantage that it disables one to go beyond the lowest order of the gradient expansion, the so-called LPA which has also been used in [27]. Although the effective average action method allows for taking into account wave-function renormalization terms in the gradient expansion, it has not been aimed in [26]. In [1] the functional RG method has been applied to the 1PI effective action for single-particle quantum mechanics using the sharp gliding momentum cut-off as control parameter of the evolution. Truncating the expansion of the 1PI effective action at quartic terms, RG flow equations for the 1PI twoand four-point vertex functions have been derived. As an example of applications, the energy of the ground state and that of the first excited state have been determined numerically. The momentum-dependence of the proper self-energy has been taken with, but the role of wave-function renormalization has not been analysed directly. The authors found in accordance with the finding in [27] that the Lehman-expansion of the propagator is dominated by the single pole corresponding to the first excited state that hints on the negligible role of the wavefunction renormalization. Our purpose is to analyse the role of wavefunction renormalization more thoroughly in terms of two different IS RG schemes, the CS type one (CSi) with the imaginary mass as control parameter and another one (ISg) with the quartic coupling as control parameter.

First, we shall apply the CSi RG scheme to the $O(N)$-symmetric generalization of the one-dimensional quartic oscillator considering the oscillator coordinate $\underline{q}=\left(q_{1}, \ldots, q_{N}\right)$ an $O(N)$ vector in the internal space. Due to $O(N)$ symmetry the anharmonic potential can only depend on powers of the $O(N)$ scalar $\underline{q}^{2}=\sum_{a=1}^{N} q_{a}^{2}=q_{a} q_{a} . O(N)$ 
symmetry couples any one-dimensional oscillator $q_{a}(a=1,2, \ldots, N)$ of the system to all of the others in a rather particular manner even if we restrict ourselves to a quartic potential, as we shall do throughout this work. We shall derive the functional CSi RG equation for the 1PI effective action, $\Gamma[q]$ being the generating functional of the 1PI Green functions of the elementary field variable $q$. In the right-hand side of the evolution equation the inverse matrix under the trace shall be Neumann-expanded in its off-diagonal piece and the terms up to the quadratic ones in the latter only kept. The solution of that truncated evolution equation shall be looked for in the next-to-leading order of the gradient expansion, including field-dependent wavefunction renormalization besides the local potential. In the case of quartic self-interaction the field-independent wavefunction renormalization emerges as a two-loop effect, therefore the way to generate it by an essentially one-loop evolution equation opens via including field-dependent wavefunction renormalization terms, as well. We shall take with only the quartic ones of those. The RG evolution of the couplings and their IR values are then numerically investigated in the function of the dimension $N$ of the oscillator. One should notice that the truncations used may lead to erroneous results for sufficiently large dimension $N$ and bare coupling $g_{B}$ [38]. We shall conclude that the $O(N)$ symmetric anharmonic oscillator with any dimension $N$ exhibits a single phase and the IR couplings including those of the wavefunction renormalization depend smoothly of the dimension $N$.

At second, we apply the ISg RG scheme to the second Legendre-transform of the generating functional of connected Green-functions of the bi-local composite operator $q_{t} q_{t^{\prime}}$, to the 2PI effective action $\Gamma[G]$ that is the functional of the single-particle propagator $G$. According to our results obtained in the CSi RG scheme no qualitative changes occur with increasing dimension $N$ in the trivial phase structure and the IR scaling laws of the anharmonic oscillator. Therefore we shall restrict our further discussion to the simple anharmonic oscillator with a single degree of freedom in the internal space, ie. with $N=1$. The $2 \mathrm{PI}$ effective action $\Gamma[G]$ takes its minimum at the propagator for the ground state and its value at that minimum determines the energy of the ground state. Parameters of the wavefunction renormalization and the excitation energy of the first excited state can be read of from the propagator of the ground state. The renormalized coupling of the quartic term of the potential is determined via the second functional derivative of the effective action $\Gamma[G]$ at the ground state, the two-particle propagator of the vacuum in quantum field-theoric terms. The RG evolution equation for the $2 \mathrm{PI}$ effective action shall be derived and solved with an Ansatz keeping the terms up to the quadratic ones in the 2-particle propagator, called expansion in the 2-particle channel (E2PC). Finally we shall compare the results obtained in second order of the bare coupling $g_{B}$ by means of the CSi and the ISg RG schemes, as well as with other results taken from the literature. The dependence of the contributions of the order $\mathcal{O}\left(g_{B}^{2}\right)$ on the RG schemes used shall be demonstrated.

The structure of the paper is as follows. Sect. II contains the derivation of the CSi RG evolution equation for the 1PI effective action for the $O(N)$ symmetric anharmonic oscillator, the derivation of the coupled set of RG equations for the running couplings present in the truncated gradient expansion of the effective action, and the comparison of the numerical solutions obtained in the independent mode approximation (IMA), the LPA, and the approximation including wavefunction renormalization (AWF). The existence of only a single phase is stressed by discussing the sensitivity of the IR couplings to their bare values, as well. Sect. III starts with the derivation of the RG evolution equation for the 2PI effective action for the one-component $(N=1)$ quartic anharmonic oscillator, that is followed by the solutions first in the IMA, and afterwards in the truncated E2PC. Finally, the results for the various couplings are determined keeping the terms up to the order $\mathcal{O}\left(g_{B}^{2}\right)$ and compared with the results obtained in the CSi RG scheme in second order perturbation expansion, as described in Appendix A 3, with the WH RG scheme in the same approximation shortly outlined in Appendix B and the well-known results of the Rayleigh-Schrödinger perturbation expansion (RS PE). In Sect. IV the conclusions are drawn. The details of the derivation of CSi RG evolution equations are given in Appendix A 1, the loop integrals needed in both schemes are given in Appendix A 2. Appendix B presents a short derivation of the WH RG equation for a one-dimensional quantum mechanical system and its application to the anharmonic oscillator in the second order of the perturbation expansion.

\section{CSI RG FOR THE N-DIMENSIONAL OSCILLATOR}

\section{A. RG equations}

In the Euclidean formulation of the quantum field-theoric models the CS RG scheme is realized by evolving the mass as a control parameter from above the UV momentum cut-off $\Lambda$ towards its physical value continuously. The evolution accounts for the quantum fluctuations gradually according to their increasing amplitude. Here we realize the similar RG procedure in the real-time formulation by introducing an evolving imaginary mass as control parameter which vanishes in the IR limit. Euclidean flow equations being easier to manage numerically are then obtained by analytic continuation of the control parameter. The advantage of the real-time approach is that the path integral remains well-defined even for a quantum system with double-well potential, ie. for negative values of the bare parameter 
$\omega_{0}^{2}$. Nevertheless one does not expect any phase transition with analytic continuation of the $\omega_{0}^{2}$ parameter to the negative real axis, since there emerges no spontaneous symmetry breaking in quantum mechanics. The large amplitude quantum fluctuations fill up the potential wells and make it convex in the IR limit (c.f. with the findings in Refs. $[26,27])$.

The basic idea is that we introduce an imaginary part $\frac{1}{2} i \mu^{2} \int_{t} \underline{q}_{t}^{2}$ into the quadratic term of the bare action $S_{B}[\underline{q}]$, in order to suppress the quantum fluctuations in the path integral for $\mu^{2} \approx \Lambda^{2}$. The real control parameter $\mu^{2}$ is then continuously decreased to zero. The imaginary quadratic part ensures the convergence of the path integral during the whole evolution. The generator functional of the connected Green-functions of the elementary 'field variable' $\underline{q}_{t}$, the time-dependent coordinate of the linear $O(N)$ symmetric anharmonic oscillator is given as

$$
S_{B}[\underline{q}]=\int_{t}\left(\frac{1}{2} \dot{q}^{2}-\frac{1}{2}\left(\omega_{0}^{2}-i \mu^{2}\right) \underline{q}^{2}-\frac{g_{B}}{24}\left(\underline{q}^{2}\right)^{2}\right) .
$$

For $\mu^{2}=\mu_{B}^{2} \approx \Lambda^{2}$, the quantum fluctuations are frozen and they can be gradually 'melted out' when the control parameter is decreased towards $\mu^{2} \rightarrow 0$. For the sake of simplicity the mass $m$ of the oscillator is set to unity. Throughout the paper we shall use the notations and conventions $\int_{t}=\int_{-T / 2}^{T / 2} d t,(T \rightarrow \infty), \int_{\omega}=\int_{-\Lambda}^{\Lambda} \frac{d \omega}{2 \pi},(\Lambda \rightarrow$ $\infty), \sum_{a=1}^{N} \int_{t} f_{a, t} g_{a, t}=\underline{f} \cdot \underline{g}, f_{a, \omega}=\int_{t} e^{i \omega t} f_{a, t}, \bar{I}_{(a, t),\left(b, t^{\prime}\right)}=\delta_{a, b} \delta_{t, t^{\prime}}=\delta_{a, b} \delta\left(t-t^{\prime}\right), \bar{I}_{(a, \omega),\left(b, \omega^{\prime}\right)}=\delta_{a, b} \delta_{\omega+\omega^{\prime}, 0}=$ $\delta_{a, b} \int_{t, t^{\prime}} e^{i \omega t+i \omega^{\prime} t^{\prime}} \delta_{t, t^{\prime}}, \delta_{\omega=0,0}=T, \operatorname{tr} A=\sum_{a=1}^{N} \int_{t, t^{\prime}} \delta_{t, t^{\prime}} A_{(a, t),\left(a, t^{\prime}\right)}=T \sum_{a=1}^{N} \int_{\omega} A_{(a, \omega),(a,-\omega)}$, and identical Latin indices denote summation like $f_{a} g_{a}=\sum_{a=1}^{N} f_{a} g_{a}$. The generating functional of the connected Green-functions of the elementary variable $\underline{q}_{t}$ is defined via the path integral

$$
e^{\frac{i}{\hbar} W[\underline{j}]}=\int \mathcal{D} \underline{q} e^{\frac{i}{\hbar} S_{B}[\underline{q}]+\frac{i}{\hbar} \underline{j} \cdot \underline{q}}
$$

in the presence of the time-local external source $\underline{j}_{t}$. The generating functional of the 1PI Green functions, the 1PI effective action as the functional of the ground-state expectation value of the coordinate, $q_{a, t}=\delta W[j] / \delta j_{a, t}$ is defined by the Legendre-transform

$$
-\Gamma[\underline{q}]=-W[\underline{j}]+\underline{j} \cdot \underline{q}
$$

for which $j_{a, t}=-\delta \Gamma[q] / \delta q_{a, t}$, and $\Gamma^{(2)} \cdot W^{(2)}=-\bar{I}$ where $W^{(2)}$ and $\Gamma^{(2)}$ stand for the second functional derivatives of the functionals $W[\bar{j}]$ and $\Gamma[\underline{q}]$, respectively. In order to find the CS-type functional evolution equation, we evaluate the partial derivative of the effective action with respect to the control parameter $\mu^{2}$,

$$
\begin{aligned}
\partial_{\mu^{2}} \Gamma[\underline{q}] & =+\partial_{\mu^{2}} W[\underline{j}]=+e^{-\frac{i}{\hbar} W[\underline{j}]} \int \mathcal{D} \underline{q} \int_{t} \frac{1}{2} i \underline{q}^{2} e^{\frac{i}{\hbar} S[\underline{q}]+\frac{i}{\hbar} \underline{j q}}=+e^{-\frac{i}{\hbar} W[\underline{j}]} \frac{1}{2} i \int_{t} \frac{\delta^{2}}{\delta \frac{i}{\hbar} j_{a, t} \delta \frac{i}{\hbar} j_{a, t}} e^{\frac{i}{\hbar} W[\underline{j}]} \\
& =+\frac{1}{2} i \int_{t}\left(\frac{\hbar}{i} \frac{\delta^{2} W[\underline{j}]}{\delta j_{a, t} \delta j_{a, t}}+\frac{\delta W[\underline{j}]}{\delta j_{a, t}} \frac{\delta W[\underline{j}]}{\delta j_{a, t}}\right)=+\frac{\hbar}{2}\left(-\operatorname{tr} \Gamma^{(2)-1}+\frac{i}{\hbar} \underline{q} \cdot \underline{q}\right) .
\end{aligned}
$$

Now we introduce the reduced effective action $\bar{\Gamma}[\underline{q}]$ with the relation $\Gamma[\underline{q}]=\bar{\Gamma}[\underline{q}]+\frac{1}{2} i \mu^{2} \underline{q} \cdot \underline{q}$ in terms of which the evolution equation takes the following form,

$$
\partial_{\mu^{2}} \bar{\Gamma}[\underline{q}]=-\frac{\hbar}{2} \operatorname{tr}\left(\bar{\Gamma}^{(2)}[\underline{q}]+i \mu^{2}\right)^{-1} .
$$

We shall look for the solution of the evolution equation in the truncated gradient expansion by making use of the Ansatz

$$
\bar{\Gamma}[\underline{q}]=-T \gamma_{\mu^{2}}+\frac{1}{2} \int_{\omega}\left(x_{\mu^{2}} \omega^{2}-\Omega_{\mu^{2}}^{2}\right) \underline{q}_{\omega} \underline{q}_{-\omega}-\int_{\omega_{1}, \ldots, \omega_{4}} v_{\mu^{2}}\left(\omega_{1}, \omega_{2}\right) \delta_{\omega_{1}+\ldots+\omega_{4}, 0} q_{a, \omega_{1}} q_{a, \omega_{2}} q_{b, \omega_{3}} q_{b, \omega_{4}}
$$

with $x_{\mu^{2}}$ for field-independent wave-function renormalization and

$$
v_{\mu^{2}}\left(\omega_{1}, \omega_{2}\right)=\frac{1}{2} \bar{y}_{\mu^{2}} \omega_{1} \omega_{2}+\frac{1}{4} \bar{Y}_{\mu^{2}}\left(\omega_{1}^{2}+\omega_{2}^{2}\right)+\frac{g}{24}=v_{\mu^{2}}\left(\omega_{2}, \omega_{1}\right)=v_{\mu^{2}}\left(-\omega_{1},-\omega_{2}\right),
$$

where the first two terms stand for the field-dependent, quadratic wavefunction renormalization,

$$
-\int_{t}\left(\frac{1}{2} \bar{y}_{\mu^{2}} \underline{q}^{2} \underline{\dot{q}}^{2}+\frac{1}{2} \bar{Y}_{\mu^{2}} \underline{q}^{2}(\underline{\ddot{q} q})\right)
$$


and the third term for the quartic self-interaction. Integrating by parts one finds

$$
-\int_{t} \ddot{q}_{a, t} q_{a, t} q_{b, t} q_{b, t}=\int_{t}\left[\dot{q}_{a, t} \dot{q}_{a, t} q_{b, t} q_{b, t}+2 \dot{q}_{a, t} q_{a, t} \dot{q}_{b, t} q_{b, t}\right]
$$

so that one can perform the replacement $\omega_{1}^{2} \Leftrightarrow\left(\omega_{1} \omega_{2}+2 \omega_{1} \omega_{3}\right)$ in the kernel of the last integral term in the right-hand side of Eq. (6) and write

$$
-\int_{t}\left(\frac{1}{2} \bar{y}_{\mu^{2}} \underline{q}^{2} \underline{\dot{q}}^{2}+\frac{1}{2} \bar{Y}_{\mu^{2}} \underline{q}^{2}(\underline{\ddot{q} q})\right)=-\int_{t}\left(\frac{1}{2} y_{\mu^{2}} \underline{q}^{2} \underline{\dot{q}}^{2}+\frac{1}{2} Y_{\mu^{2}}(\underline{\dot{q} q})^{2}\right)
$$

$y_{\mu^{2}}=\bar{y}_{\mu^{2}}-\bar{Y}_{\mu^{2}}$ and $Y_{\mu^{2}}=-2 \bar{Y}_{\mu^{2}}$.

Let us separate off the diagonal piece $\bar{G}^{-1}$ and the off-diagonal piece $A$ of the matrix

$$
\bar{\Gamma}^{(2)}[\underline{q}]+i \mu^{2}=\bar{G}^{-1}+A
$$

where $\bar{G}_{(c, \omega),\left(d, \omega^{\prime}\right)}=G_{\omega} \delta_{c, d} \delta_{\omega+\omega^{\prime}, 0}$ with $G_{\omega}=\left[x_{\mu^{2}} \omega^{2}-\Omega_{\mu^{2}}^{2}+i \mu^{2}\right]^{-1}$ and

$$
A_{(c, \omega),\left(d, \omega^{\prime}\right)}=-\frac{\delta^{2}}{\delta q_{c,-\omega} \delta q_{d,-\omega^{\prime}}} \int_{\omega_{1}, \ldots, \omega_{4}} \delta_{\omega_{1}+\ldots+\omega_{4}, 0} v_{\mu^{2}}\left(\omega_{3}, \omega_{4}\right) q_{a, \omega_{1}} q_{a, \omega_{2}} q_{b, \omega_{3}} q_{b, \omega_{4}}=\delta_{c, d} B_{\omega, \omega^{\prime}}+\bar{C}_{(c, \omega),\left(d, \omega^{\prime}\right)}
$$

with

$$
\begin{aligned}
B_{\omega, \omega^{\prime}} & =-2 \int_{\omega_{1}, \omega_{2}} \delta_{-\omega-\omega^{\prime}+\omega_{1}+\omega_{2}, 0}\left[v_{\mu^{2}}\left(\omega_{1}, \omega_{2}\right)+v_{\mu^{2}}\left(\omega, \omega^{\prime}\right)\right] q_{a, \omega_{1}} q_{a, \omega_{2}}=B_{\omega^{\prime}, \omega} \\
\bar{C}_{(c, \omega),\left(d, \omega^{\prime}\right)} & =-4 \int_{\omega_{1}, \omega_{2}} \delta_{-\omega-\omega^{\prime}+\omega_{1}+\omega_{2}, 0}\left[v_{\mu^{2}}\left(\omega_{1},-\omega\right)+v_{\mu^{2}}\left(\omega_{2},-\omega^{\prime}\right)\right] q_{c, \omega_{1}} q_{d, \omega_{2}}=\bar{C}_{\left(d, \omega^{\prime}\right),(c, \omega)} .
\end{aligned}
$$

As to the next we Neumann-expand the inverse matrix in the right-hand side of the evolution equation (5), keeping the terms up to the second order in the off-diagonal piece,

$$
\begin{aligned}
-\frac{\hbar}{2} \operatorname{tr}\left[\bar{G}^{-1}+A\right]^{-1} & =-\frac{\hbar}{2} \operatorname{tr}[\bar{G}-\bar{G} A \bar{G}+\bar{G} A \bar{G} A \bar{G}-+\ldots] \\
& =-\frac{N \hbar}{2} \int_{\omega} G_{\omega}+\frac{\hbar}{2} \int_{\omega} G_{\omega}^{2} A_{(a, \omega),(a,-\omega)}-\frac{\hbar}{2} \int_{\omega, \omega^{\prime}} G_{\omega}^{2} A_{(c, \omega),\left(d, \omega^{\prime}\right)} G_{\omega^{\prime}} A_{\left(d,-\omega^{\prime}\right),(c,-\omega)}-+\ldots
\end{aligned}
$$

It is worthwhile noticing here that for $N=1$ the terms for field-dependent wavefunction renormalization given in Eqs. (8) or (10) merge into a single one,

$$
-\int_{\omega_{1}, \ldots, \omega_{4}} \delta_{\omega_{1}+\ldots+\omega_{4}, 0} \frac{1}{2} \Upsilon_{\mu^{2}} \omega_{1} \omega_{2} q_{\omega_{1}} q_{\omega_{2}} q_{\omega_{3}} q_{\omega_{4}}
$$

with $\Upsilon_{\mu^{2}}=\bar{y}_{\mu^{2}}-3 \bar{Y}_{\mu^{2}}$. It is illuminating to give the graphical representation of the first few terms of the Neumannexpansion in the functional CSi equation (5) for the case $N=1$, shown in Fig. 1.

The details of the evaluation of the integrals in the right-hand side of Eq. (5) are given in Appendix A 1. In addition to the truncation of the Neumann-expansion we have expanded each term of the Neumann-series in powers of the Fourier-transform $q_{a, \omega}$ of the field variable as well as in powers of its frequency index $\omega$ and neglected the higher-order terms absent in Ansatz (6). In order to perform the expansion in the frequency (i.e. in the time-derivative of the field variable) we used the expansion of the propagator,

$$
G_{\omega+\alpha}=G_{\omega}-2 x_{\mu^{2}} \omega G_{\omega}^{2} \alpha-\left[1-4 x_{\mu^{2}} \omega^{2} G_{\omega}\right] x_{\mu^{2}} G_{\omega}^{2} \alpha^{2}+\mathcal{O}\left(\alpha^{3}\right) .
$$

Furthermore, we have to make use of identity (9) in order to transform all derivative terms into one of the forms present in Ansatz (6). The truncation the Neumann-expansion and that of the gradient expansion may lead to erroneous results in the IR scaling regime for sufficiently strong coupling $g_{B}$ and/or large number $N$ of dimensions. The steps described above enable one to express the trace in the right-hand side of the evolution equation (5) in terms of the loop integrals

$$
I_{n, s}\left(\mu^{2}, \Omega_{\mu^{2}}^{2}\right)=\hbar \int_{-\Lambda}^{\Lambda} \frac{d \omega}{2 \pi} G_{\omega}^{n} \omega^{s}
$$




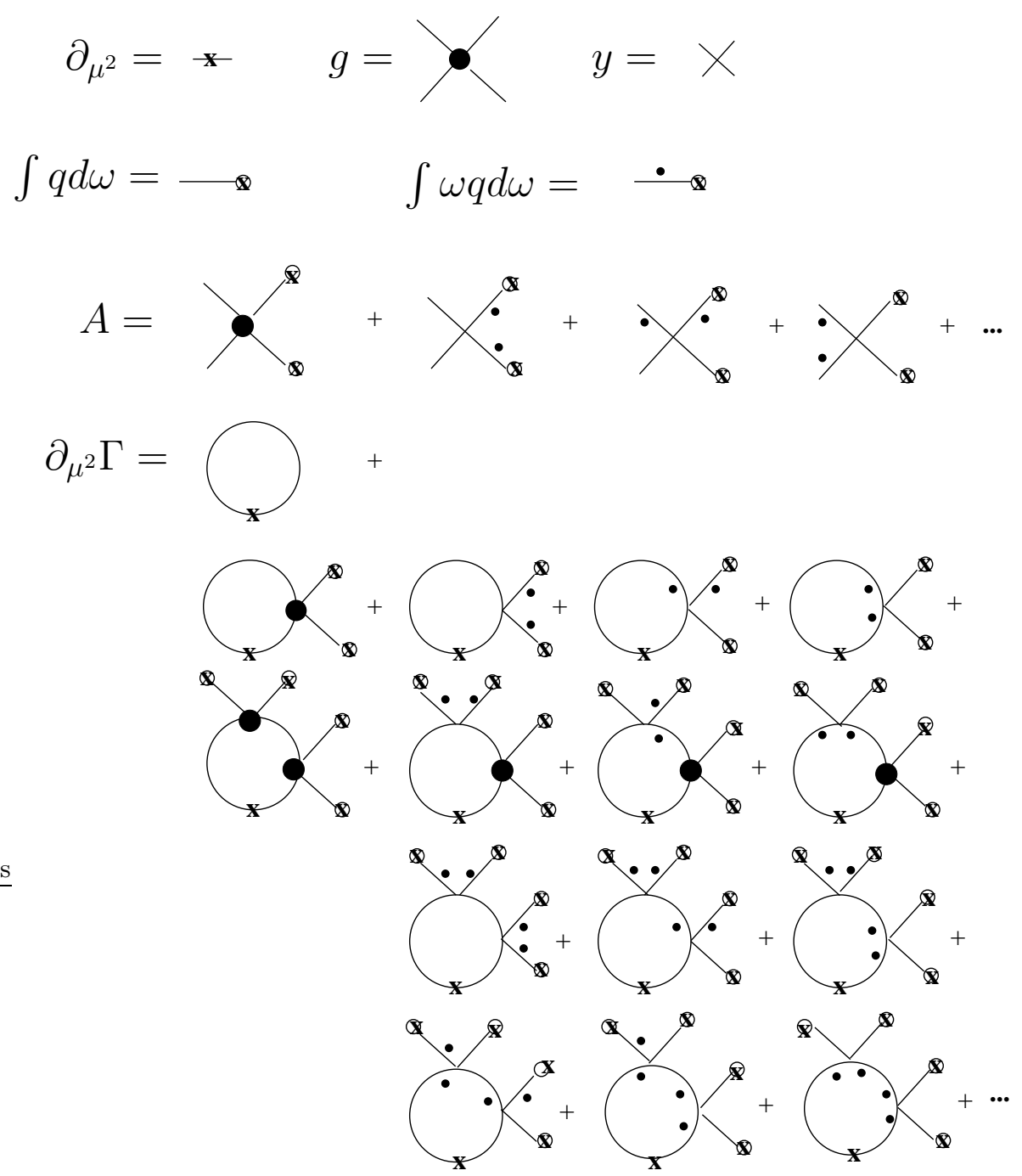

FIG. 1: Feynman diagrams for the vertices and the right-hand side of the evolution equation in $\mathrm{CSi}$ RG for the $N=1$ dimensional oscillator.

and find the evolution equation

$$
\begin{aligned}
& -T \partial_{\mu^{2}} \gamma_{\mu^{2}}+\frac{1}{2} \int_{\omega_{1}}\left(\partial_{\mu^{2}} x_{\mu^{2}} \omega_{1}^{2}-\partial_{\mu^{2}} \Omega_{\mu^{2}}^{2}\right) q_{a, \omega_{1}} q_{a,-\omega_{1}} \\
& -\int_{\omega_{1}, \ldots, \omega_{4}} \delta_{\omega_{1}+\ldots+\omega_{4}, 0}\left(\frac{1}{2} \partial_{\mu^{2}} \bar{y}_{\mu^{2}} \omega_{1} \omega_{2}+\frac{1}{2} \partial_{\mu^{2}} \bar{Y}_{\mu^{2}} \omega_{1}^{2}+\frac{1}{24} \partial_{\mu^{2}} g\right) q_{a, \omega_{1}} q_{a, \omega_{2}} q_{b, \omega_{3}} q_{b, \omega_{4}} \\
= & -\frac{T N}{2} I_{1,0}+\frac{1}{2}\left[N \bar{y}_{\mu^{2}}-(N+2) \bar{Y}_{\mu^{2}}\right] I_{2,0} \int_{\omega_{1}} \omega_{1}^{2} q_{a, \omega_{1}} q_{a,-\omega_{1}} \\
& +\frac{1}{2}\left(\left[N \bar{y}_{\mu^{2}}-(N+2) \bar{Y}_{\mu^{2}}\right] I_{2,2}-\frac{(N+2) g_{\mu^{2}}}{6} I_{2,0}\right) \int_{\omega_{1}} q_{a, \omega_{1}} q_{a,-\omega_{1}}+T_{1}+T_{2}+T_{3}
\end{aligned}
$$

where the purely quartic terms $T_{i}(i=1,2,3)$ are given by Eqs. (A9)-(A11). In the limit $\Lambda \rightarrow \infty$ the loop-integrals can be taken analytically, and all expressed in terms of a few ones, $I_{n, 0}$ with $n=1,2,3,4$, (see Appendix A2). Comparing the coefficients of the corresponding terms of the gradient expansion in both sides of Eq. (18), one arrives at the RG evolution equations for the couplings in the our approximation, called previously AWF,

$$
\partial_{\mu^{2}} \gamma=\frac{N}{2} I_{1,0}
$$




$$
\begin{gathered}
\partial_{\mu^{2}} \Omega^{2}=-[N \bar{y}-(N+2) \bar{Y}] I_{2,2}+\frac{(N+2) g}{6} I_{2,0}, \\
\partial_{\mu^{2}} g=12\left[N \bar{y}^{2}+2(N+2) \bar{y} \bar{Y}+(N+8) \bar{Y}^{2}\right] I_{3,4}-4 g[(N+2) \bar{y}-(N+8) \bar{Y}] I_{3,2}+\frac{(N+8) g^{2}}{3} I_{3,0}, \\
\partial_{\mu^{2}} x=[N \bar{y}-(N+2) \bar{Y}] I_{2,0}, \\
\partial_{\mu^{2}} \bar{y}=8 x^{2}\left[N \bar{y}^{2}-2(N+2) \bar{y} \bar{Y}+(N+2) \bar{Y}^{2}\right] I_{5,6}-\frac{8(N+2) g}{3} x^{2}(\bar{y}-\bar{Y}) I_{5,4}+\frac{2(N+2) g^{2}}{9} x^{2} I_{5,2} \\
-10 x\left[N \bar{y}^{2}-2(N+2) \bar{y} \bar{Y}+(N+2) \bar{Y}^{2}\right] I_{4,4}+2(N+2) g x(\bar{y}-\bar{Y}) I_{4,2}-\frac{(N+2) g^{2}}{18} x I_{4,0} \\
+4\left[-2 \bar{y}^{2}-(N+2) \bar{y} \bar{Y}+(N+2) \bar{Y}^{2}\right] I_{3,2}+\frac{(N+2) g}{3}(\bar{y}+\bar{Y}) I_{3,0}, \\
\partial_{\mu^{2}} \bar{Y}=8 x^{2}\left[N \bar{y}^{2}-2(N+2) \bar{y} \bar{Y}+(N+6) \bar{Y}^{2}\right] I_{5,6}-\frac{8 g}{3} x^{2}[(N+2) \bar{y}-(N+6) \bar{Y}] I_{5,4}+\frac{2(N+6) g^{2}}{9} x^{2} I_{5,2} \\
-10 x\left[N \bar{y}^{2}-2(N+2) \bar{y} \bar{Y}+(N+6) \bar{Y}^{2}\right] I_{4,4}+2 g x[(N+2) \bar{y}-(N+6) \bar{Y}] I_{4,2}-\frac{(N+6) g^{2}}{18} x I_{4,0} \\
+\left[2 N \bar{y}^{2}-8(N+3) \bar{y} \bar{Y}+(6 N+40) \bar{Y}^{2}\right] I_{3,2}-\frac{2 g}{3}[2 \bar{y}-(N+7) \bar{Y}] I_{3,0} .
\end{gathered}
$$

For the sake of simplicity, we have suppressed the lower index $\mu^{2}$ of the running couplings. The loop-integrals occurring in these equations are UV finite except of $I_{1,0}$, so that the IR limits of all couplings can be determined for $\Lambda \rightarrow \infty$ with exception of the constant term of the 1PI effective action determining the energy of the ground state. The $x$-dependence of the right-hand sides of the evolution equations is partly explicit, partly implicit occurring via the propagators in the loop-integrals. The evolution of the field-independent wavefunction renormalization $x$ is generated via the evolution of the field-dependent wavefunction renormalization parametrized by $\bar{y}$ and $\bar{Y}$ (c.f. Eq. $(22)$ ) because those start to evolve already at the UV scales due to the terms containing the loop-integrals $I_{5,2}$ and $I_{4,0}$ in the right-hand sides of Eqs. (23) and (24) even for vanishing bare values $\bar{y}_{B}=\bar{Y}_{B}=0$. The evolution equations for the LPA can easily be obtained by setting $x=1$ and $\bar{y}=\bar{Y}=0$ identically. It is worthwhile noticing that the analytic continuation of the RG equations to Euclidean space via the replacement $-\boldsymbol{i} \boldsymbol{\mu}^{\mathbf{2}} \rightarrow \lambda$ for real $\lambda$ decreasing from $\lambda_{B} \approx \Lambda^{2}$ towards the IR limit $\lambda \rightarrow 0$ is rather suitable for the numerical treatment since the Euclidean RG flow does not develop imaginary parts for the couplings.

For $N=1$ only the single coupling $\Upsilon=\bar{y}-3 \bar{Y}$ for field-dependent wavefunction renormalization occurs and the $\mathrm{RG}$ equations (19)-(24) reduce to the following ones,

$$
\begin{gathered}
\partial_{\mu^{2}} \gamma=\frac{1}{2} I_{1,0}, \\
\partial_{\mu^{2}} \Omega^{2}=-\Upsilon I_{2,2}+\frac{1}{2} g I_{2,0}, \\
\partial_{\mu^{2}} g=12 \Upsilon^{2} I_{3,4}-12 g \Upsilon I_{3,2}+3 g^{2} I_{3,0}, \\
\partial_{\mu^{2}} x=\Upsilon I_{2,0}, \\
\partial_{\mu^{2}} \Upsilon=-16 x^{2} \Upsilon^{2} I_{5,6}+16 g x^{2} \Upsilon I_{5,4}-4 g^{2} x^{2} I_{5,2}+20 \Upsilon^{2} x I_{4,4}-12 g x \Upsilon I_{4,2}+g^{2} x I_{4,0}-14 \Upsilon^{2} I_{3,2}+5 g \Upsilon I_{3,0} .
\end{gathered}
$$

We see now for $N=1$ that the parameter $x$ starts to evolve in the UV scaling regime because of the parameter $\Upsilon$ does due to the nonvanishing terms $-4 g^{2} x^{2} I_{5,2}+g^{2} x I_{4,0}$ in the right-hand side of Eq. (29). 


\section{B. Solution of the CSi RG equations}

\section{IMA}

The IMA corresponds to the replacement of the running couplings by their bare values $\Omega_{B}^{2}=\omega_{0}^{2}, g_{B}, x_{B}=1$, $\bar{y}_{B}=\bar{Y}_{B}=0$ into the right-hand sides of the evolution equations (25)-(29). Making use of the loop integrals of Appendix A 2 in the limit $\Lambda \rightarrow \infty$ and the analytic continuation to the Euclidean space via the replacement $\lambda=-\boldsymbol{i} \boldsymbol{\mu}^{\mathbf{2}}$, one can easily integrate Eqs. (25)-(29) from the initial scale $\lambda_{B} \rightarrow \infty$ down to the gliding scale $\lambda$. The UV divergence of the ground-state energy can be removed by the choice $\gamma_{B}=\frac{1}{2} N \hbar\left[\omega_{0}^{2}+\lambda_{B}\right]^{\frac{1}{2}}$. The UV scaling laws obtained in that manner can then be extrapolated to the IR limit $\lambda \rightarrow 0$ :

$$
\begin{aligned}
& \gamma_{0}=\frac{1}{2} N \hbar \omega_{0}, \quad \Omega_{0}^{2}=\omega_{0}^{2}\left[1+\frac{4(N+2) \xi}{3}\right], \quad g_{0}=g_{B}\left[1-\frac{2(N+8) \xi}{3}\right], \\
& x_{0}=1, \quad \hbar \bar{y}_{0}=-\frac{4(N+2) \omega_{0} \xi^{2}}{9}, \quad \hbar \bar{Y}_{0}=-\frac{4(N+6) \omega_{0} \xi^{2}}{9},
\end{aligned}
$$

where the dimensionless parameter $\xi=\frac{g_{B} \hbar}{16 \omega_{0}^{3}}$ of the Rayleigh-Schrödinger perturbation expansion [39] has been introduced. We see that the one-loop corrections of the couplings depend linearly on the dimension $N$. The energy of the ground state $E_{0}=\gamma_{0}=\frac{1}{2} N \hbar \omega_{0}$ is just the sum of the zero-point energies of the number $N$ of independent harmonic oscillators in this approximation, the one-loop result. The energy gap between the first excited state and the ground state, i.e. in field-theoric terms the minimum energy of the one-particle excitations, $E_{1}-E_{0}=\hbar \sqrt{\Omega_{0}^{2} / x_{0}}=\hbar \omega_{0} \sqrt{1+4(N+2)(\xi / 3)}$ increases with increasing bare coupling strength $g_{B}$ and number of dimension $N$. The extrapolated IR value of the quartic coupling $g_{0}$ decreases with its increasing $g_{B}$ and $N$ in the IMA. Although there occurs a field-dependent wavefunction renormalization of the order $\mathcal{O}\left(g_{B}^{2}\right)$ in the IMA, the field-independent wavefunction renormalization is absent since it is a two-loop effect in the lowest nonvanishing order of the loop expansion. The beta-functions do not change sign, so that one can conclude that the extrapolation of the UV scaling laws to the IR region seems to predict only a single phase of the quantum oscillator independently of its dimension $N$. However, restricting ourselves to that approximation, we would get in trouble with the change of sign of $g_{0}$ for sufficiently large $g_{B}$ and $N$, as well as with a continuation of these formulae to negative values of $\omega_{0}^{2}$ corresponding to a symmetric double-well bare potential.

\section{Numerical integration of the CSi RG equations}

The numerical integration of the RG equations (20)-(24) obtained in the CSi RG scheme in the AWF and analytically continued to the Euclidean space has been performed by means of a fourth-order Runge-Kutta algorithm. In Figs. 2 and 3 the RG flow obtained in AWF is compared to RG flows obtained in the IMA and the LPA. (The latter is obtained by setting $x=1, \bar{y}=\bar{Y}=0$ identically.) One can see, that the flows in the various approximations are qualitatively the same, i.e. after the UV scaling region both the dimensionful frequency $\Omega^{2}$ and the quartic coupling $g$ saturate at constant values in the IR region. The UV scaling is described properly by all approximations, but they give different values in the IR limit. The IMA corresponding to the one-loop approximation gives the largest value for $\Omega^{2}$ and the smallest one for $g$. More reliable results are obtained in the LPA and AWF which resum infinitely many Feynman diagrams. Both of the Figs. 2 and 3 clearly show that the inclusion of the wavefunction renormalization modifies only slightly the evolution as compared to the LPA. This is a consequence of the fact that the pole of the propagator, corresponding to the first excited state of the oscillator dominates in its Lehman-expansion, as discussed in $[27]$.

The flows in the AWF have common features that can be recognized from Figs. 2-5. Since the couplings $\bar{y}$ and $\bar{Y}$ scale quite similarly, we have plotted their combination $\Upsilon$ into which they merge in the case of the one-dimensional oscillator. After an UV scaling region extending roughly over three orders of magnitude of the scale parameter $\lambda$, the flow saturates in the IR region for any of the dimensionful couplings, so that all couplings are IR relevant. Namely, they tend to nonvanishing constant values in the IR limit and only a single phase is detected. The latter corresponds to the general expectation because there is no room for spontaneous symmetry breaking in the $0+1$ dimensional quantum system.

It has been found that the flows for any of the couplings do not alter qualitatively with increasing $N$. That is illustrated in Figs. 4 and 5 for the parameters of the wavefunction renormalization. The $N$-dependence of the various couplings in the IR limit is depicted in Fig. 6 for the various parameters. In this respect the IMA turned out to be misleading. According to the IMA the quantum fluctuations in the IR limit result in additive contributions to the 


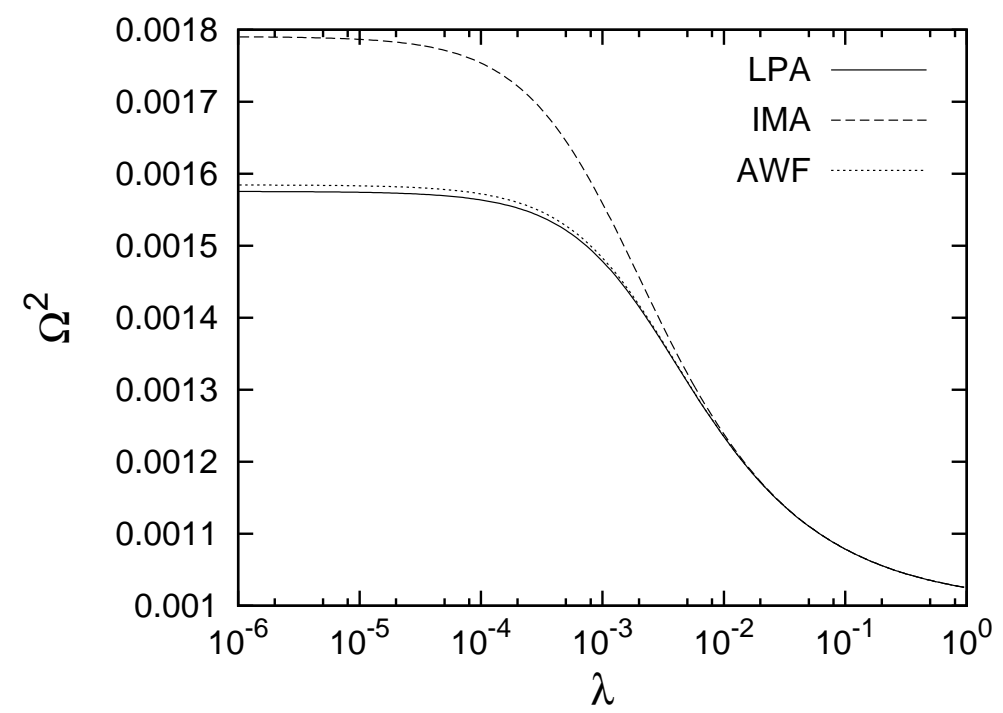

FIG. 2: The flow of the frequency parameter $\Omega^{2}$ in different approximations, for $\Omega_{B}^{2}=0.00102, g_{B}=0.0001, x_{B}=1$, $\bar{y}_{B}=\bar{Y}_{B}=0$, and $N=1$.

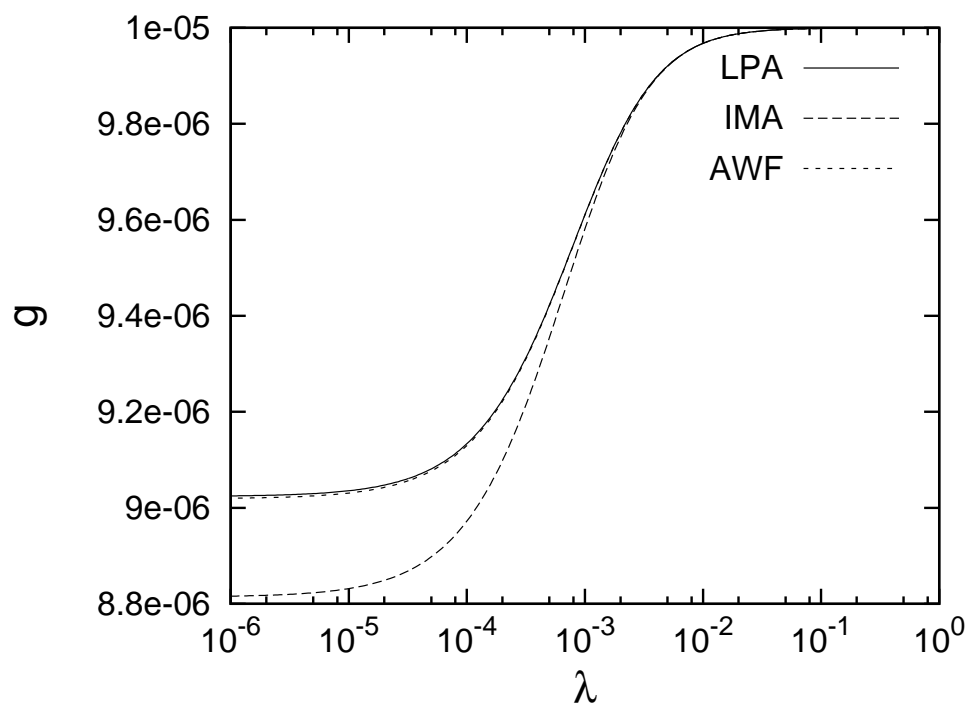

FIG. 3: The flow of the quartic coupling $g$ in different approximations, for $\Omega_{B}^{2}=0.00102, g_{B}=0.00001, x_{B}=1, \bar{y}_{B}=\bar{Y}_{B}=0$, and $N=1$.

bare values of the couplings, which are linear in the dimension $N$, but the AWF gives completely different behavior. We see in Fig. 6 that the parameter $\Omega_{0}^{2}$ shows up a powerlike dependence on $N$ for sufficiently large dimensions, $\Omega_{0}^{2} \propto N^{b_{\Omega}}$ with $b_{\Omega} \approx 0.58$. In field-theoric terms the oscillator gets more 'massive' with increasing dimension $N$. The coupling $g_{0}$ depends rather weakly on $N$ having a maximum at around $N \approx 30$. The slow decrease for large $N$ values may be the consequence of the truncations used. The neglection of higher-order monomials in the gradient expansion can cause a failure when one or both of the dimension $N$ and the bare quartic coupling $g_{B}$ are too large. It is easy to notice from the explicit form of the RG evolution equations in the IMA that the loop-expansion goes effectively with $N g_{B} \hbar$ for large $N$, so that the higher-order terms of the local potential can grow up to the same order of magnitude as the quadratic and quartic terms are when the flow reaches the border of the UV scaling region and that may result in their saturation in the IR scaling region at values of the same order of magnitude, too. In Refs. $[26,27]$ the authors take with the higher-order monoms of the local potential, as well. They have found that the renormalized coupling $g_{0}$ increases strictly monotonically with increasing bare parameter $g_{B}$ for the one-dimensional oscillator. Similarly, $g_{0}$ should increase with the dimension $N$ of the oscillator also strictly monotonically, because the the loop-expansion goes effectively with $N g_{B}$ for large $N$. As one can see in the inset of Fig. 6, the deviation 


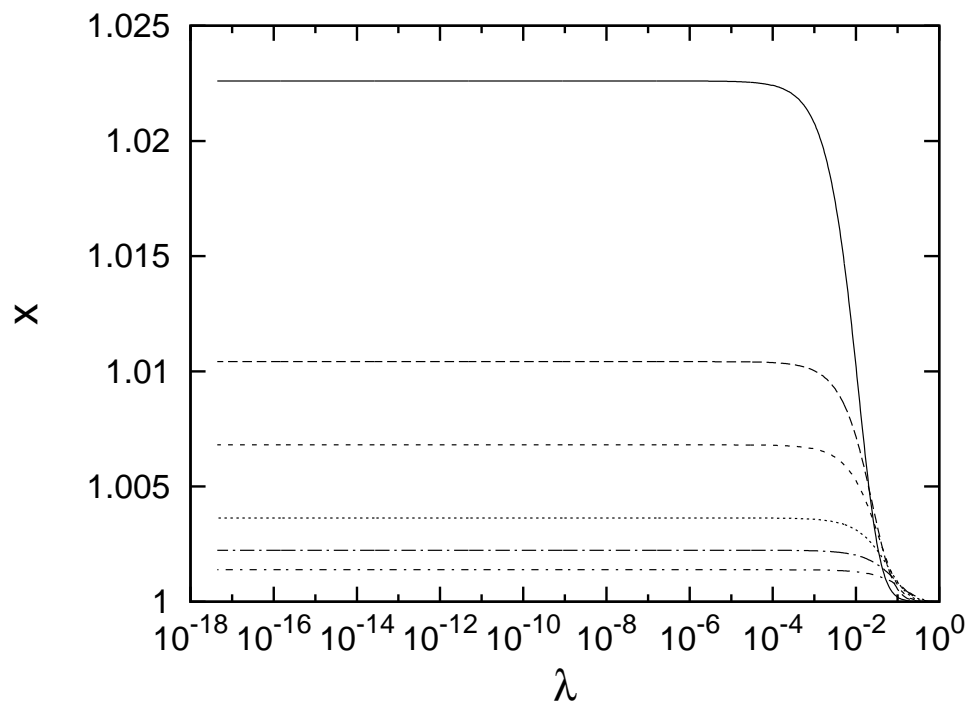

FIG. 4: The scale dependence of $x$ for $\Omega_{B}^{2}=0.001, g_{B}=0.01$, and $\bar{y}=\bar{Y}=0$. The order of the curves from the top correspond to the various values $N=2,10,20,50,100,200$, respectively.

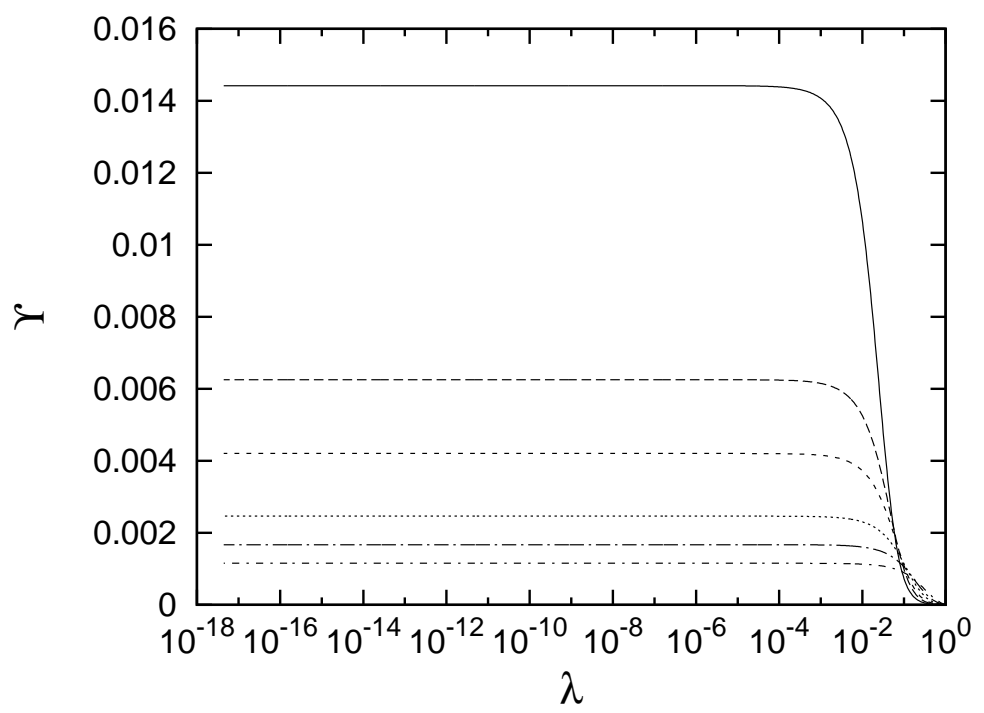

FIG. 5: The scale dependence of $\Upsilon$ for $\Omega_{B}^{2}=0.001, g_{B}=0.01$, and $\bar{y}=\bar{Y}=0$. The order of the curves from the top correspond to the various values $N=1,10,20,50,100,200$, respectively.

$x_{0}-1$ of the field-independent wavefunction renormalization parameter $x_{0}$ from its bare value as well as the coupling $\Upsilon_{0}$ of the field-dependent wavefunction renormalization go to zero according to the power laws, $x_{0}-1 \sim N^{-b_{x}}$ and $\Upsilon_{0} \sim N^{-b_{\Upsilon}}$ with $b_{x} \approx 0.55$ and $b_{\Upsilon} \approx 0.45$, respectively for increasing $N$. Therefore the wavefunction renormalization effects seem to be washed out in the limit $N \rightarrow \infty$ because of the increment of the mass gap.

The dependence of the IR parameters on the bare ones can be characterized by the sensitivity matrix, which is defined by

$$
S_{s_{1}, s_{2}}(r)=\frac{\partial s_{1}}{\partial s_{2}}
$$

where $s_{1}=\Omega^{2}, g, x, \Upsilon$ are the running couplings and $s_{2}=\Omega_{B}^{2}, g_{B}, x_{B}, \Upsilon_{B}$, and $N$ are the bare parameters and the dimension, respectively. The elements of the sensitivity matrix integrate the infinitesimal changes of the infinitesimal blocking steps during the RG flow when the ratio $r=\lambda / \lambda_{B}$ of the moving scale to the UV scale glides from $r=1$ to the IR limit $r=0$. Therefore, their values in the IR limit, $S_{s_{1}, s_{2}}(0)$ as characteristics of the global 


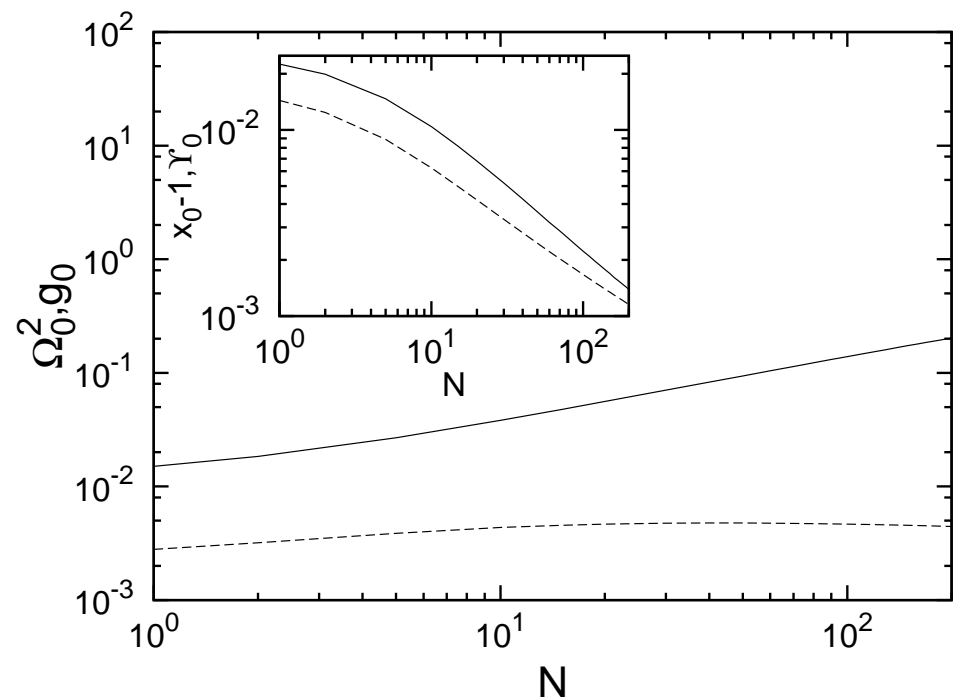

FIG. 6: The $N$-dependence of the IR values $\Omega_{0}^{2}$ (full line) and $g_{0}$ (dashed line) for $\Omega_{B}^{2}=0.001, g_{B}=0.01$, and $\bar{y}=\bar{Y}=0$. The IR values of the wavefunction renormalization parameters $x_{0}$ (full line) and $\Upsilon_{0}$ (dashed line) are shown vs. $N$ in the inset.

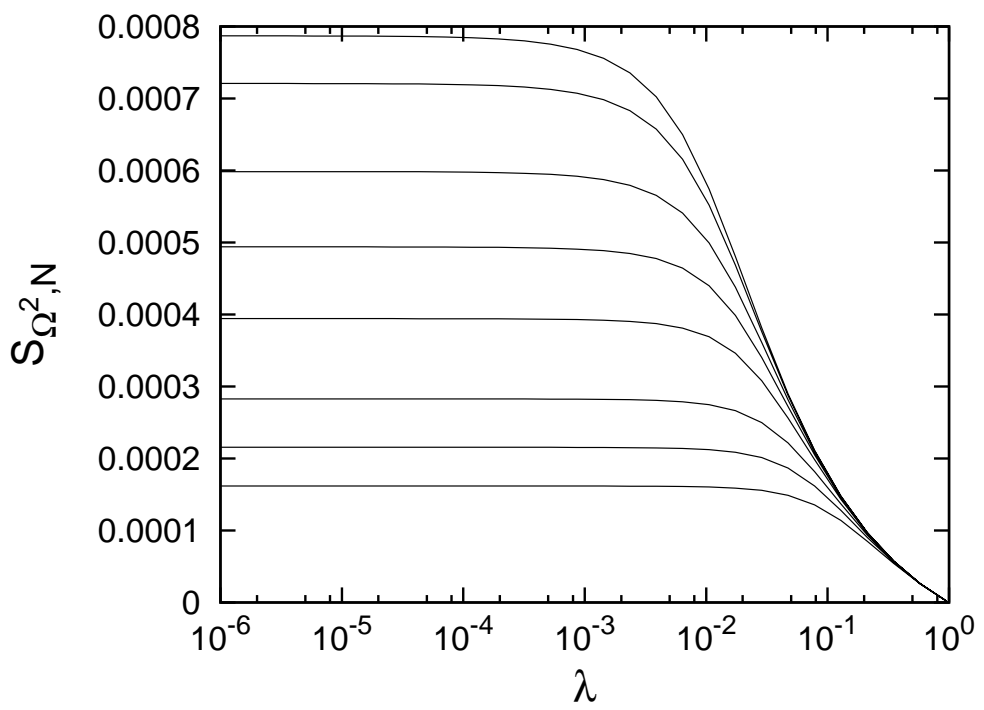

FIG. 7: The flow of the sensitivity matrix element $S_{\Omega^{2}, N}$ for $\Omega_{B}^{2}=0.001, g_{B}=0.00001$, and $\bar{y}_{B}=\bar{Y}_{B}=0$. The curves from above correspond to $N=1,2,5,10,20,50,100,200$, respectively.

RG flow may show up singular dependences on the bare parameters, which may reveal themselves in qualitatively different scale-dependences $S_{s_{1}, s_{2}}(r)$ along the various RG trajectories started in various regions of the space of the bare couplings [41]. Since the model in our case has a single symmetric phase, no such singularities are expected to occur in the sensitivity matrix. This is demonstrated in Figs. 7 and 8, where the matrix elements $S_{\Omega^{2}, N}$ and $S_{\Omega^{2}, \Omega_{B}^{2}}$ are shown to vary monotonically with decreasing scale $\lambda$. The flow of these matrix elements do not alter its character with increasing $N$ (illustrated in Fig. 7). The UV and the IR scaling regions are again clearly distinguishable and the flow saturates in the IR region. The larger is the dimension $N$, the weaker is the sensitivity of the IR parameter $\Omega_{0}^{2}$ to the same small increment $\Delta N$ of the dimension.

Quite similarly, the character of the scale-dependences of $S_{\Omega^{2}, N}(r)$ and $S_{\Omega^{2}, \Omega_{B}^{2}}(r)$ do not alter starting the RG flow with various bare values $\Omega_{B}^{2}$ (see Fig. 8), including also its negative values corresponding to a double-well bare potential. This is another signal that the anharmonic oscillator exhibits only a single, symmetric phase. As it was thoroughly discussed in $[26,27]$ the large-amplitude, low-momentum quantum fluctuations fill up the minima of the double-well potential and one ends up with a convex potential and a unique ground state in the IR limit. It was also 


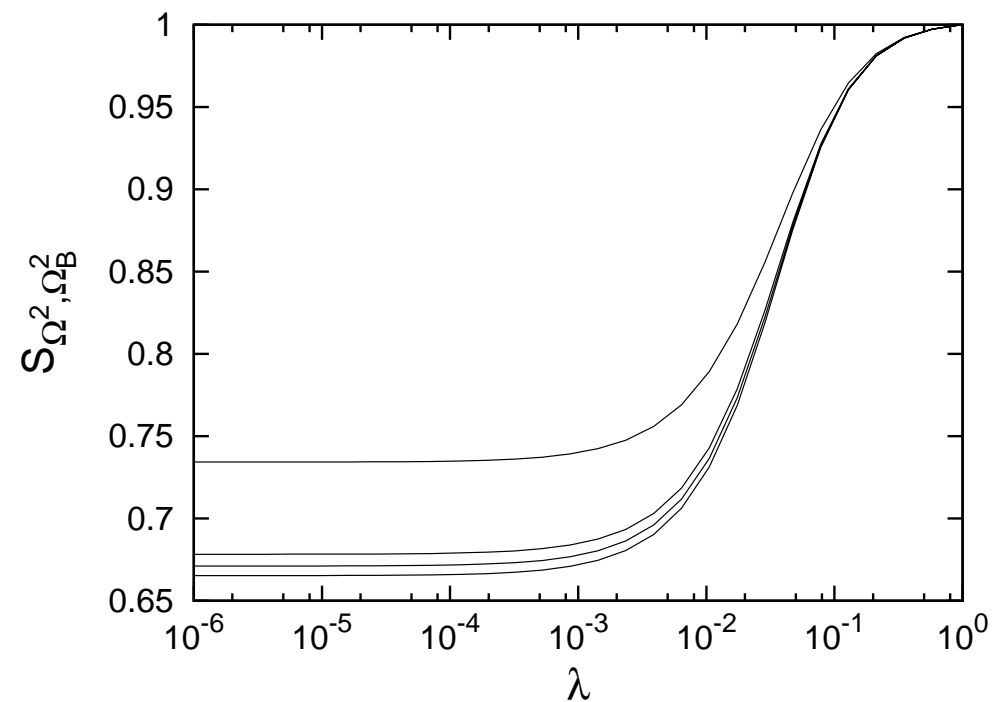

FIG. 8: The flow of the sensitivity matrix element $S_{\Omega^{2}, \Omega_{B}^{2}}$ for $N=50$ and $g_{B}=0.00001$. The curves from below correspond to $\Omega_{B}^{2}=-0.001,-0.0001,0.001,0.01$, respectively.

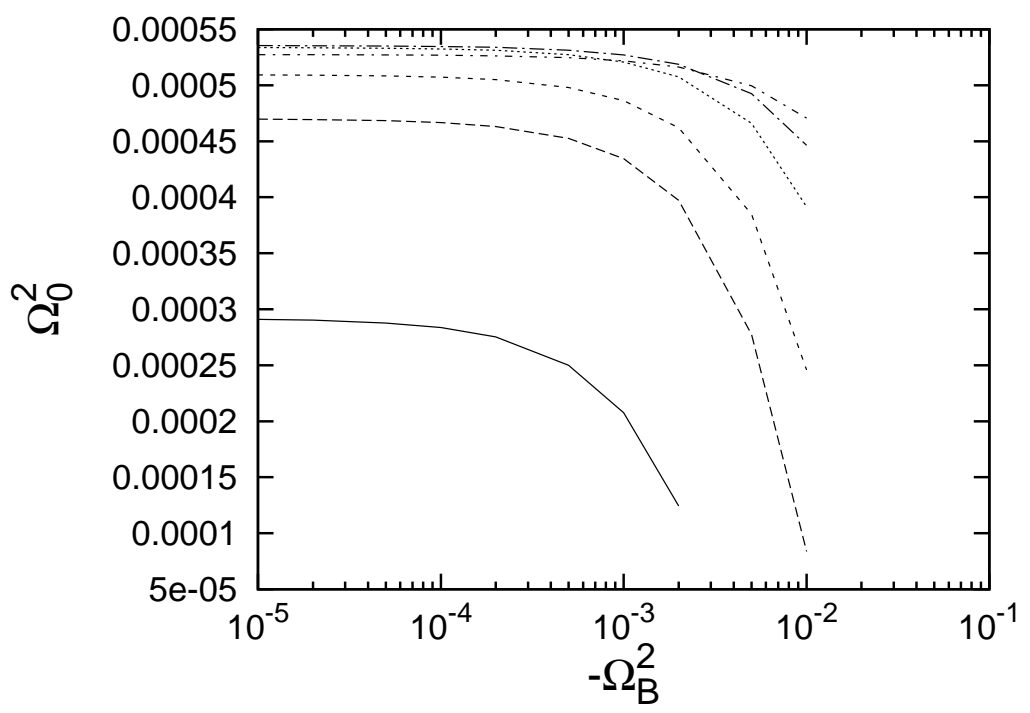

FIG. 9: The $N$-dependence of the IR values $\Omega_{0}^{2}$ vs. the bare parameter $\Omega_{B}^{2}$. The curves from below correspond to $N=$ $1,10,20,50,100,200$, respectively.

discussed that for sufficiently small bare quartic coupling the RG evolution equations show up singularities and one has to take into account time-dependent saddle point configurations for evaluating the path integral, that has been achieved by turning to an instanton gas approximation. In Fig. 9 we illustrate that the RG flow started with negative parameter values $\Omega_{B}^{2}<0$ ends up with some positive values $\Omega_{0}^{2}$ in the IR limit. We note that in our case the evolution gets wrong for too large negative UV values of $\Omega_{B}^{2}$ due to the strong truncation of the local potential in the ansatz (6). As $N$ is increased we get larger and larger IR frequency values $\Omega_{0}^{2}$ according to the power-law established previously.

Closing the discussion of the numerical results obtained in the CSi RG scheme, we can make an estimate of the spatial extension of the ground-state wavefunction. As shown in the Appendix A 4, the variance of the coordinate operator $q$ can be estimated by making use of the Thomas-Reiche-Kuhn sum rule [40] and the dominance of the first pole in the Lehmann-expansion of the single-particle propagator [27]. With the help of the scaling of the renormalized coupling with increasing dimension $N$ established above, we get from (A37) that the variance scales as

$$
\left\langle 0\left|\underline{q}^{2}\right| 0\right\rangle \approx \frac{N x^{3 / 2} \hbar}{2 \Omega} \sim N^{1-\frac{1}{2} b_{\Omega}} \sim N^{0.71} .
$$


This means that with increasing dimension $N$ the quantum oscillator becomes more and more delocalized because of the increasing the number of degrees of freedom faster than that of the 'mass', ie. the frequency $\Omega_{0}$ of the oscillator.

\section{ISG RG FOR THE ONE-DIMENSIONAL OSCILLATOR}

\section{A. Evolution equations}

Further on we shall restrict ourselves to the study of the one-dimensional anharmonic oscillator, since the scaling laws for the $N$-dimensional anharmonic oscillator do not show up any significant qualitative modification in dependence on the number of dimensions $N$ according to our findings in the framework of the CSi RG scheme. Now we choose an IS RG scheme in that the bare coupling $g_{B}$ of the anharmonic quartic interaction term acts as control parameter and it is evolved from zero towards a final positive value. For the sake of simplicity, below we shall suppress its index and denote the control parameter of the evolution simply by $g$. Here we apply the ISg RG scheme to the so-called second Legendre-transform, the Legendre-transform of the generating functional $W[J]$ of the connected Green-functions of the bilocal operator $q_{t} q_{t^{\prime}}$ defined via the path integral

$$
e^{\frac{i}{\hbar} W[J]}=\int \mathcal{D} q e^{\frac{i}{\hbar} \int_{t}\left[\frac{1}{2} \dot{q}^{2}-\frac{1}{2}\left(\omega_{0}^{2}-i \epsilon\right) q^{2}-\frac{g}{24} q^{4}\right]+\frac{i}{\hbar} \frac{1}{2} \int_{t, t^{\prime}} q_{t} J_{t, t^{\prime}} q_{t^{\prime}}}
$$

with the symmetric external bi-local source $J_{t, t^{\prime}}=J_{t^{\prime}, t}[35]$. We have introduced the infinitesimal imaginary piece of the quadratic term of the potential by the replacement $\omega_{0}^{2} \rightarrow \omega_{0}^{2}-i \epsilon$ in order to make the path integral convergent. In the presence of the external source $J$ the propagator $G$ is given as

$$
W_{t, t^{\prime}}^{(1)}=\frac{\delta W[J]}{\delta \frac{1}{2} J_{t, t^{\prime}}}=\left\langle T\left(q_{t} q_{t^{\prime}}\right)\right\rangle=G_{t, t^{\prime}}, \quad W_{\omega, \omega^{\prime}}^{(1)}=\frac{\delta W[J]}{\delta \frac{1}{2} J_{-\omega,-\omega^{\prime}}}=G_{\omega, \omega^{\prime}}
$$

The vanishing source $J=0$ corresponds to the ground state with the propagator $\left\langle T\left(q_{t} q_{t^{\prime}}\right)\right\rangle_{J=0}=G_{\text {gr }} t, t^{\prime}$. The second Legendre-transform, the $2 \mathrm{PI}$ effective action $\Gamma[G]$ is defined as

$$
\Gamma[G]=-W[J]+\frac{1}{2} \operatorname{tr}\left(G^{\operatorname{tr}} \cdot J\right)
$$

and the relations

$$
\frac{\delta \Gamma[G]}{\delta G_{t, t^{\prime}}}=\frac{1}{2} J_{t, t^{\prime}}, \quad \frac{\delta \Gamma[G]}{\delta G_{\omega, \omega^{\prime}}}=\frac{1}{2} J_{-\omega,-\omega^{\prime}}
$$

hold. The symmetry of the source $J$ implies the symmetry $G_{t, t^{\prime}}=G_{t^{\prime}, t}$ of the propagator. The second functional derivatives satisfy the identity

$$
I=W^{(2)}: \Gamma^{(2)}, \quad W^{(2)}=\Gamma^{(2)-1 I}=\Gamma^{(2)-1}: I
$$

in shorthand notations with $A: B=\int_{t, t^{\prime}} A_{(., .),\left(t, t^{\prime}\right)} B_{\left(t, t^{\prime}\right),(., .)}=\int_{\omega, \omega^{\prime}} A_{(., .),\left(\omega, \omega^{\prime}\right)} B_{\left(-\omega,-\omega^{\prime}\right),(., .)}$. For later convenience we introduce the matrices $I, L$, and $\mathbb{1}$,

$$
\begin{aligned}
& I_{\left(t_{3}, t_{4}\right),\left(t_{1}, t_{2}\right)}=\frac{1}{2}\left(\delta_{t_{1}, t_{3}} \delta_{t_{2}, t_{4}}+\delta_{t_{1}, t_{4}} \delta_{t_{2}, t_{3}}\right), \quad I_{\left(\omega_{3}, \omega_{4}\right),\left(\omega_{1}, \omega_{2}\right)}=\frac{1}{2}\left(\delta_{\omega_{1}+\omega_{3}, 0} \delta_{\omega_{2}+\omega_{4}, 0}+\delta_{\omega_{1}+\omega_{4}, 0} \delta_{\omega_{2}+\omega_{3}, 0}\right), \\
& L_{\left(t_{3}, t_{4}\right),\left(t_{1}, t_{2}\right)}=\delta_{t_{1}, t_{2}} \delta_{t_{1}, t_{3}} \delta_{t_{1}, t_{4}}, \quad L_{\left(\omega_{3}, \omega_{4}\right),\left(\omega_{1}, \omega_{2}\right)}=\delta_{\omega_{1}+\omega_{2}+\omega_{3}+\omega_{4}, 0}, \\
& \mathbb{1}_{\left(t_{3}, t_{4}\right),\left(t_{1}, t_{2}\right)}=\delta_{t_{1}, t_{3}} \delta_{t_{2}, t_{4}}, \quad \mathbb{1}_{\left(\omega_{3}, \omega_{4}\right),\left(\omega_{1}, \omega_{2}\right)}=\delta_{\omega_{1}+\omega_{3}, 0} \delta_{\omega_{2}+\omega_{4}, 0} .
\end{aligned}
$$

The matrix $I$ acts over the two-particle (in field-theoric sense) subspace as the projector on the symmetrical subspace (that of the two-particle states being symmetric under the exchange of the particles) and plays the role of the identity operator in that subspace and $L: I=L$ holds, while $\mathbb{1}$ represents the usual identity operator over the entire twoparticle subspace. The inverse $A^{-1}$ of an arbitrary two-particle matrix $A$ satisfies $A^{-1}: A=\mathbb{1}$, while for the inverse $A^{-1 I}=A^{-1}: I$ of the same matrix in the symmetrical subspace $A^{-1 I}: A=I$ holds. Further on let us denote the trace of an arbitrary two-particle matrix by $\operatorname{Tr} A=\int_{t, t^{\prime}} A_{\left(t, t^{\prime}\right),\left(t, t^{\prime}\right)}=\int_{\omega, \omega^{\prime}} A_{\left(\omega, \omega^{\prime}\right),\left(-\omega,-\omega^{\prime}\right)}$.

The bare coupling $g$ is turned on gradually from zero to some finite value in order to control the evolution of the 2PI effective action satisfying the following RG evolution equation,

$$
\begin{aligned}
\partial_{g} \Gamma[G] & =-\partial_{g} W[J]=\frac{1}{24} e^{-\frac{i}{\hbar} W[J]} \int \mathcal{D} q \int_{t} q_{t}^{4} e^{\frac{i}{\hbar} \int_{t}\left(\frac{1}{2} \dot{q}^{2}-\frac{1}{2}\left(\omega_{0}^{2}-i \epsilon\right) q^{2}-\frac{g}{24} q^{4}\right)+\frac{i}{\hbar} \frac{1}{2} q \cdot J \cdot q} \\
& =\frac{1}{24}\left(\frac{\hbar}{i}\right)^{2}\left[\frac{i}{\hbar} \operatorname{Tr}\left(L: W^{(2)}\right)+\left(\frac{i}{\hbar}\right)^{2} W^{(1)}: L: W^{(1)}\right]=\frac{1}{24} \frac{\hbar}{i}\left[\operatorname{Tr}\left(L: \Gamma^{(2)-1 I}\right)+\frac{i}{\hbar} G: L: G\right] .
\end{aligned}
$$


Separating off the trivial term controlling the RG evolution, one can introduce the reduced 2PI effective action $\bar{\Gamma}[G]$ via the relation

$$
\Gamma[G]=\bar{\Gamma}[G]+\frac{g}{24} G: L: G
$$

and recast Eq. (39) in the form

$$
\partial_{g} \bar{\Gamma}[G]=\frac{1}{24} \frac{\hbar}{i} \operatorname{Tr}\left[\left(\bar{\Gamma}^{(2)}[G]+\frac{g}{12} L\right)^{-1 I}: L\right]
$$

\section{B. Solution of the evolution equation}

\section{IMA}

In order to make a guess on the functional form of the reduced $2 \mathrm{PI}$ effective action $\bar{\Gamma}[G]$ for the linear quartic oscillator, we evaluate it first in the tree-approximation, and solve the RG equation in the IMA. The tree-level approximation corresponds to the vanishing value of the control parameter, $g=0$, ie. the case of the linear harmonic oscillator, for which the Gaussian path integral can be evaluated in a straightforward manner, yielding

$$
W^{\text {tree }}[J]=\frac{i \hbar}{2} \operatorname{tr} \ln \left[\left(\omega^{2}-\omega_{0}^{2}+i \epsilon\right) \bar{I}+J\right]
$$

and

$$
\Gamma^{\mathrm{tree}}[G]=\frac{i \hbar}{2} \operatorname{tr} \ln G-\frac{1}{2} \operatorname{tr}\left[\left(\omega^{2}-\omega_{0}^{2}+i \epsilon\right) \bar{I} \cdot G\right]+\text { const. }=\bar{\Gamma}^{\text {tree }}[G]
$$

up to an UV divergent additive constant (being independent of the bare parameters). The necessary condition of the extremum of the $2 \mathrm{PI}$ effective action in the tree-approximation, $\delta \bar{\Gamma}^{\mathrm{IMA}}[G] / \delta G=0$ provides the propagator in the ground state of the linear harmonic oscillator,

$$
G_{\mathrm{gr}}^{\mathrm{tree}}=+i \hbar\left[\left(\omega^{2}-\omega_{0}^{2}+i \epsilon\right)\right]^{-1} \bar{I}
$$

as expected. The inverse of the noninteracting two-particle propagator is given via

$$
\mathcal{G}_{\left(-\omega_{3},-\omega_{4}\right),\left(-\omega_{1}, \omega_{2}\right)}^{\text {tree-1I}}[G]=\bar{\Gamma}_{\left(-\omega_{1},-\omega_{2}\right),\left(-\omega_{3},-\omega_{4}\right)}^{\mathrm{tree}(2)}[G]=-\frac{i \hbar}{4}\left[G_{-\omega_{3},-\omega_{1}}^{-1} G_{-\omega_{2},-\omega_{4}}^{-1}+G_{-\omega_{3},-\omega_{2}}^{-1} G_{-\omega_{1},-\omega_{4}}^{-1}\right] .
$$

Hence one finds

$$
\mathcal{G}_{\left(\omega_{3}, \omega_{4}\right),\left(\omega_{1}, \omega_{2}\right)}^{\text {tree }}[G]=\frac{i}{\hbar}\left(G_{\omega_{3}, \omega_{1}} G_{\omega_{4}, \omega_{2}}+G_{\omega_{4}, \omega_{1}} G_{\omega_{3}, \omega_{2}}\right)
$$

that we write as $\mathcal{G}^{\text {tree }}=\frac{i}{\hbar}(G G)^{I}$ introducing the shorthand notations $(A B)_{(c, d),(a, b)}^{I}=\frac{1}{2}\left(A_{c, a} B_{d, b}+A_{d, a} B_{c, b}\right)$, and for later use $\alpha:: \beta=\int_{a, \ldots, d} \alpha_{[\ldots],[(c, d),(a, b)]} \beta_{[(-c,-d),(-a,-b)],[\ldots]}$ where Latin indices stand for frequency indices. For later use we also evaluate the first and second functional derivatives of $\mathcal{G}^{\text {tree }}[G]$,

$$
\begin{aligned}
& \frac{\delta \mathcal{G}_{\left(\omega_{3}, \omega_{4}\right),\left(\omega_{1}, \omega_{2}\right)}^{\text {tree }}[G]}{\delta G_{-\omega,-\omega^{\prime}}}=\frac{i}{\hbar} \frac{1}{2}\left(\left[\delta_{\omega_{3}+\omega, 0} \delta_{\omega_{1}+\omega^{\prime}, 0} G_{\omega_{4}, \omega_{2}}+\delta_{\omega_{4}+\omega, 0} \delta_{\omega_{2}+\omega^{\prime}, 0} G_{\omega_{3}, \omega_{1}}+\left(\omega_{4} \Leftrightarrow \omega_{3}\right)\right]+\left(\omega \Leftrightarrow \omega^{\prime}\right)\right), \\
& \frac{\delta^{2} \mathcal{G}_{\left(\omega_{3}, \omega_{4}\right),\left(\omega_{1}, \omega_{2}\right)}^{\text {tree }}[G]}{\delta G_{-\omega,-\omega^{\prime}} \delta G_{-\omega^{\prime \prime},-\omega^{\prime \prime \prime}}}= \frac{i}{4 \hbar}\left[\left(\left[\delta_{\omega_{3}+\omega, 0} \delta_{\omega_{1}+\omega^{\prime}, 0} \delta_{\omega_{4}+\omega^{\prime \prime}, 0} \delta_{\omega_{2}+\omega^{\prime \prime \prime}, 0}+\delta_{\omega_{4}+\omega, 0} \delta_{\omega_{2}+\omega^{\prime}, 0} \delta_{\omega_{3}+\omega^{\prime \prime}, 0} \delta_{\omega_{1}+\omega^{\prime \prime \prime}, 0}\right.\right.\right. \\
&\left.\left.\left.+\left(\omega_{4} \Leftrightarrow \omega_{3}\right)\right]+\left(\omega \Leftrightarrow \omega^{\prime}\right)\right)+\left(\omega^{\prime \prime} \Leftrightarrow \omega^{\prime \prime \prime}\right)\right] .
\end{aligned}
$$

In order to obtain the $2 \mathrm{PI}$ effective action in the IMA, we have to insert the tree-level expression $\bar{\Gamma}^{\text {tree }}[G]$ into the right-hand side of the evolution equation (41) and integrate it over the control parameter from the initial value $g=0$ 
to some finite value $g$. Then we get

$$
\begin{aligned}
\bar{\Gamma}^{\mathrm{IMA}}[G] & =\bar{\Gamma}^{\text {tree }}[G]+\frac{1}{24} \frac{\hbar}{i} \int_{0}^{g} d \bar{g} \operatorname{Tr}\left[\left(\bar{\Gamma}^{\operatorname{tree}(2)}[G]+\frac{\bar{g}}{12} L\right)^{-1 I}: L\right]=\bar{\Gamma}^{\operatorname{tree}}[G]-\frac{i \hbar}{2} \operatorname{Tr}\left[I: \ln \left(\mathbb{1}+\frac{g}{12} L: \mathcal{G}^{\text {tree }}[G]\right)\right] \\
& \left.=\bar{\Gamma}^{\text {tree }}[G]+\frac{i \hbar}{2} \sum_{n=1}^{\infty} \frac{(-1)^{n}}{n}\left(\frac{g}{12}\right)^{n} \operatorname{tr}\left(L: \mathcal{G}^{\text {tree }}[G]\right)^{n}\right] .
\end{aligned}
$$

The inverse propagator of the ground state in the IMA is given through the necessary condition of the extremum of the 2PI effective action $\Gamma^{\mathrm{IMA}}[G]$, ie. $\delta \Gamma^{\mathrm{IMA}}[G] / \delta G=0$, that yields the equation for the propagator of the ground state in the IMA, $G_{\mathrm{gr}}^{\mathrm{IMA}}$,

$$
G_{\mathrm{gr}}^{\mathrm{IMA}-1}=G_{\mathrm{gr}}^{\mathrm{tree}-1}+\frac{g}{12} \operatorname{Tr}\left[L: \frac{\delta \mathcal{G}^{\mathrm{tree}}[G]}{\delta G}:\left(\mathbb{1}+\frac{g}{12} L: \mathcal{G}^{\mathrm{tree}}[G]\right)^{-1}\right]_{G=G_{\mathrm{gr}}^{\mathrm{IMA}}}+\frac{i g}{6 \hbar}\left(L: G_{\mathrm{gr}}^{\mathrm{IMA}}\right),
$$

ie.

$$
\begin{aligned}
G_{\mathrm{gr} \omega, \omega^{\prime}}^{\mathrm{IMA}-1}= & G_{\mathrm{gr} \omega, \omega^{\prime}}^{\mathrm{tree}-1}+\left[\frac{i g}{2 \hbar} \int_{\omega_{1}, \omega_{2}} \delta_{\omega+\omega^{\prime}+\omega_{1}+\omega_{2}, 0} G_{\omega_{1}, \omega_{2}}\right. \\
& \left.+\frac{g^{2}}{18 \hbar^{2}} \int_{\omega_{1}, \omega_{2}, \ldots, \omega_{6}} \delta_{\omega+\omega_{1}+\omega_{3}+\omega_{4}, 0} \delta_{\omega^{\prime}+\omega_{2}+\omega_{5}+\omega_{6}, 0} G_{\omega_{1}, \omega_{2}} G_{\omega_{3}, \omega_{5}} G_{\omega_{4}, \omega_{6}}+\mathcal{O}\left(g^{3}\right)\right]_{G=G_{\mathrm{gr}}^{\mathrm{IMA}}}
\end{aligned}
$$

when the terms up to the order $\mathcal{O}\left(g^{2}\right)$ are only kept. Multiplying both sides of this equation by $G_{\mathrm{gr}}^{\mathrm{IMA}}$ from the left and $G_{\mathrm{gr}}^{\text {tree }}$ from the right, one would obtain the Schwinger-Dyson equation in the IMA for the propagator of the ground state, but for our later purposes it is more convenient to retain the present form of the equation. The solution of Eq. (51), the propagator of the ground state can be used to determine the energy of the ground state,

$$
E_{0}=\lim _{T \rightarrow \infty} \frac{1}{T} \Gamma^{\mathrm{IMA}}\left[G_{\mathrm{gr}}^{\mathrm{IMA}}\right]
$$

and the 2PI 4-point vertex function,

$$
\begin{aligned}
& \gamma_{\left(\omega_{3}, \omega_{4}\right),\left(\omega_{1}, \omega_{2}\right)}^{[2]}=\mathcal{G}_{\left(\omega_{3}, \omega_{4}\right),\left(\omega_{1}, \omega_{2}\right)}^{\mathrm{IMA}-1}\left[G_{\mathrm{gr}}^{\mathrm{IMA}}\right]-\mathcal{G}_{\left(\omega_{3}, \omega_{4}\right),\left(\omega_{1}, \omega_{2}\right)}^{\mathrm{tree}}\left[G_{\mathrm{gr}}^{\mathrm{IMA}}\right] \\
& =\frac{g}{4} \delta_{\omega_{1}+\omega_{2}+\omega_{3}+\omega_{4}, 0}-\frac{i g^{2}}{24 \hbar} \int_{\omega_{1}^{\prime}, \omega_{2}^{\prime}, \omega_{3}^{\prime}, \omega_{4}^{\prime}}\left[\delta_{\omega_{1}+\omega_{3}+\omega_{1}^{\prime}+\omega_{3}^{\prime}, 0} \delta_{\omega_{2}+\omega_{4}+\omega_{2}^{\prime}+\omega_{4}^{\prime}, 0}+\left(\omega_{1} \Leftrightarrow \omega_{2}\right)\right] G_{\mathrm{gr} \omega_{1}^{\prime}, \omega_{2}^{\prime}}^{\mathrm{IMA}} G_{\mathrm{gr} \omega_{3}^{\prime}, \omega_{4}^{\prime}}^{\mathrm{IMA}}+\mathcal{O}\left(g^{3}\right)
\end{aligned}
$$

with $\mathcal{G}^{\mathrm{IMA}-1 \mathrm{I}}[G]=\Gamma^{\mathrm{IMA}(2)}[G]$.

The evolution equation (41) allows for a simple diagrammatic picture, shown in Fig. 10. The single vertex without the factor $g$ (empty circle) present in each of the diagrams for $\partial_{g} \bar{\Gamma}$ occurs due to the partial derivative with respect to $g$. The result of the IMA is the resummation of the ring diagrams shown for $\Gamma[G]-\Gamma^{\text {tree }}[G]$ in the fourth and fifth lines in Fig. 10. In the fourth line we depicted the expansion of the 2PI effective action in powers of the tree-level two-particle

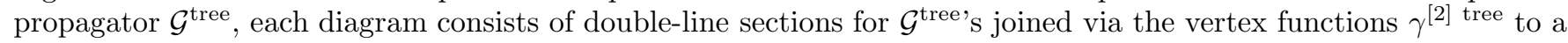
closed loop. As one can see from the expansion in powers of the propagator $G$ shown in the fifth line, the 2PI vertex function is taken at the tree-level, $\gamma^{[2]}$ tree $=g L / 12$. The proper self-energy insertion $\Sigma^{\text {IMA }}=G^{-1 \text { IMA }}-G^{-1 \text { tree }}$ and the 2PI 4-point vertex function $\gamma^{[2]}$ IMA in the IMA are depicted in Fig. 11.

\section{Expansion in the 2-particle channel (E2PC)}

The functional dependence of the reduced 2PI effective action $\bar{\Gamma}^{\mathrm{IMA}}[G]$ on the tree-level 2-particle propagator $\mathcal{G}^{\text {tree }}$ in the IMA brings one to the idea to look for a better approximation of the effective action as an expansion in powers of the tree-level 2-particle propagator $\mathcal{G}^{\text {tree }}=2 \frac{i}{\hbar}(G G)^{I}$ making the Ansatz

$$
\bar{\Gamma}^{\mathrm{E} 2 \mathrm{PC}}[G]=\Gamma^{\mathrm{tree}}[G]+\frac{1}{2} \bar{\gamma}^{[2]}::(G G)^{I}+\frac{1}{24}(G G)^{I}:: \gamma^{[4]}::(G G)^{I}+\ldots,
$$




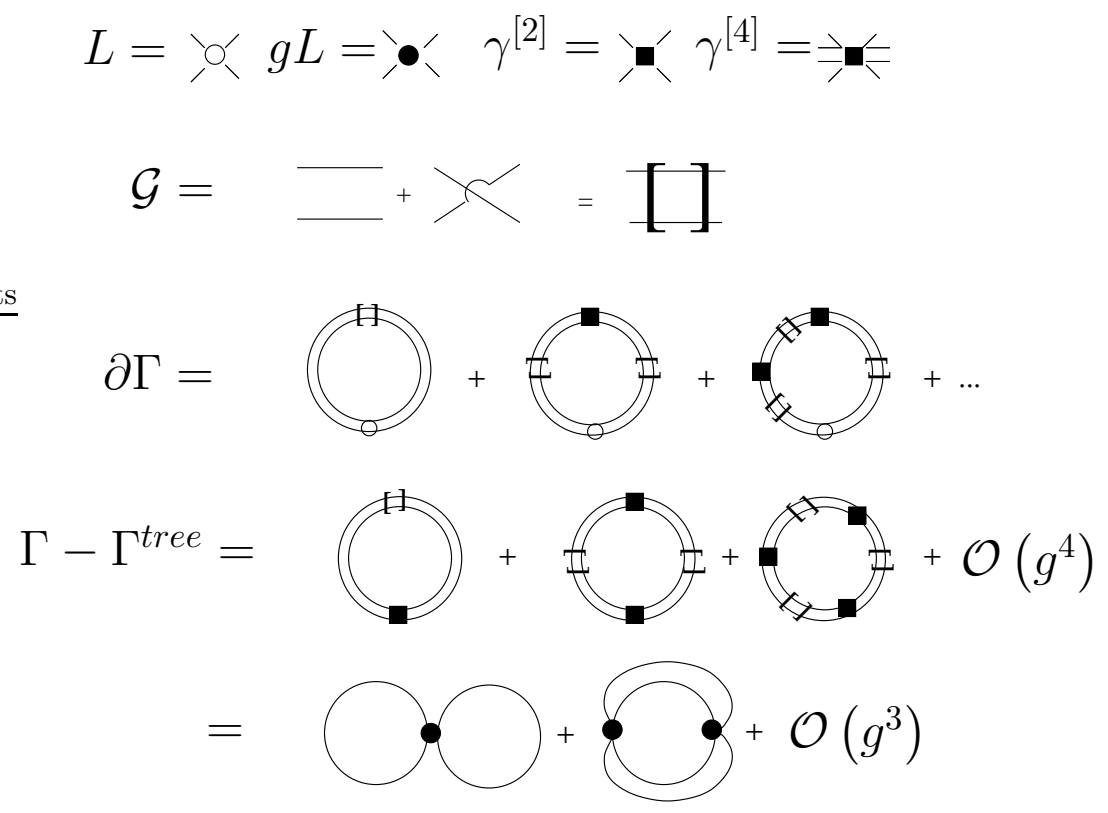

FIG. 10: Feynman diagrams for the RG evolution of the 2PI effective action in the IMA.

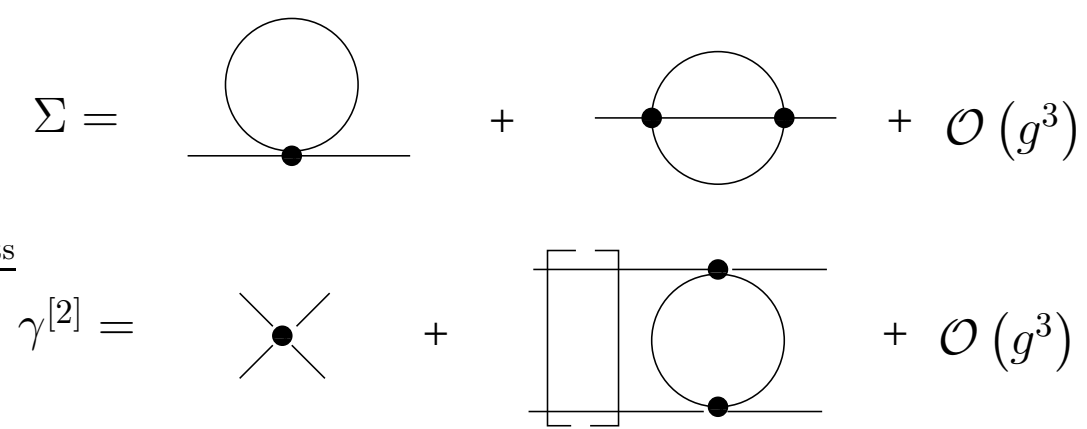

FIG. 11: Feynman diagrams for the proper self-energy insertion $\Sigma=G^{-1 \text { IMA }}-G^{-1 \text { tree }}$ and the 2 PI vertex function $\gamma^{[2]}$ IMA .

where the symmetry properties

$$
\begin{aligned}
& \bar{\gamma}_{(a, b),(c, d)}^{[2]}=\bar{\gamma}_{(b, a),(c, d)}^{[2]}=\bar{\gamma}_{(c, d),(a, b)}^{[2]}, \\
& \gamma_{[(a, b),(c, d)],\left[\left(a^{\prime}, b^{\prime}\right),\left(c^{\prime}, d^{\prime}\right)\right]}^{[4]}=\gamma_{[(b, a),(c, d)],\left[\left(a^{\prime}, b^{\prime}\right),\left(c^{\prime}, d^{\prime}\right)\right]}^{[4]}=\gamma_{[(c, d),(a, b)],\left[\left(a^{\prime}, b^{\prime}\right),\left(c^{\prime}, d^{\prime}\right)\right]}^{[4]}=\gamma_{\left[\left(a^{\prime}, b^{\prime}\right),\left(c^{\prime}, d^{\prime}\right)\right],[(a, b),(c, d)]}^{[4]}
\end{aligned}
$$

are required (Latin indices stand for frequency indices) for the 2PI vertex functions, $\bar{\gamma}^{[2]}$ and $\gamma^{[4]}$. We shall solve the evolution equation (41) inserting the Ansatz (54) into it, expanding its right-hand side in Neumann-series, and keeping the terms up to the quartic ones in $G$, ie. those of the order $\mathcal{O}\left(\left[(G G)^{I}\right]^{2}\right)$ in its both sides. The approximation with the E2PC goes beyond the IMA when the inverse of the 2-particle propagator, $\bar{\Gamma}^{(2)}$ has been replaced by $\Gamma^{\operatorname{tree}(2)}=\mathcal{G}^{\text {tree-1}}[G]$ in the right-hand side of the RG equation (41). Now the Ansatz (54) in powers of $\mathcal{G}^{\text {tree }}[G]$ implies

$$
\bar{\Gamma}^{\mathrm{E} 2 \mathrm{PC}(2)}[G]=\mathcal{G}^{\text {tree-1}}[G]+\bar{\gamma}^{[2]}+\mathcal{O}\left((G G)^{I}\right) .
$$

Having expanded the inverse matrix, ie. 2-particle propagator in the right-hand side of (41) in Neumann-series as

$$
\mathcal{G}^{\mathrm{E} 2 \mathrm{PC}}[G]=\Gamma^{\mathrm{E} 2 \mathrm{PC}(2)-1}[G]=\left(\bar{\Gamma}^{\mathrm{E} 2 \mathrm{PC}(2)}[G]+\frac{g}{12} L\right)^{-1}=\mathcal{G}^{\text {tree }}[G]-\mathcal{G}^{\text {tree }}[G]: \gamma^{[2]}: \mathcal{G}^{\text {tree }}[G]+\mathcal{O}\left(\left[(G G)^{I}\right]^{3}\right)
$$

with the 2PI 4-point vertex function,

$$
\gamma^{[2]}=\Gamma^{(2)}[G]-\mathcal{G}^{\text {tree-1}}[G]=\bar{\gamma}^{[2]}+\frac{g}{12} L,
$$


we obtained the following coupled set of RG evolution equations for the 2PI 4-point and 8-point vertex functions,

$$
\begin{aligned}
& \partial_{g} \bar{\gamma}_{\left(\omega_{1}, \omega_{2}\right),\left(\omega_{3}, \omega_{4}\right)}^{[2]}=\frac{1}{6} L_{\left(\omega_{3}, \omega_{4}\right),\left(\omega_{1}, \omega_{2}\right)}, \\
& \partial_{g} \gamma_{\left[\left(\omega_{1}, \omega_{2}\right),\left(\omega_{3}, \omega_{4}\right)\right],\left[\left(\omega_{1}^{\prime}, \omega_{2}^{\prime}\right),\left(\omega_{3}^{\prime}, \omega_{4}^{\prime}\right)\right]}^{[4]}=-\frac{i}{\hbar}\left[\left(\left(I: \gamma^{[2]}: I\right)_{\left(\omega_{3}, \omega_{4}\right),\left(\omega_{3}^{\prime}, \omega_{4}^{\prime}\right)} L_{\left(\omega_{1}, \omega_{2}\right),\left(\omega_{1}^{\prime}, \omega_{2}^{\prime}\right)}\right.\right. \\
& \left.\left.+L_{\left(\omega_{3}, \omega_{4}\right),\left(\omega_{3}^{\prime}, \omega_{4}^{\prime}\right)}\left(I: \gamma^{[2]}: I\right)_{\left(\omega_{1}, \omega_{2}\right),\left(\omega_{1}^{\prime}, \omega_{2}^{\prime}\right)}\right)+\left(\omega_{1}, \omega_{2}\right) \Leftrightarrow\left(\omega_{3}, \omega_{4}\right)\right] .
\end{aligned}
$$

Those can be integrated straightforwardly for the initial conditions $\bar{\gamma}_{g=0}^{[2]}=0, \gamma_{g=0}^{[4]}=0$, and one gets

$$
\begin{aligned}
\bar{\gamma}_{\left(\omega_{1}, \omega_{2}\right),\left(\omega_{3}, \omega_{4}\right)}^{[2]} & =\frac{g}{6} L_{\left(\omega_{1}, \omega_{2}\right),\left(\omega_{3}, \omega_{4}\right)} \\
\gamma_{\left[\left(\omega_{1}, \omega_{2}\right),\left(\omega_{3}, \omega_{4}\right)\right],\left[\left(\omega_{1}^{\prime}, \omega_{2}^{\prime}\right),\left(\omega_{3}^{\prime}, \omega_{4}^{\prime}\right)\right]}^{[4]} & =-\frac{i g^{2}}{4 \hbar}\left[\delta_{\omega_{1}+\omega_{2}+\omega_{1}^{\prime}+\omega_{2}^{\prime}, 0} \delta_{\omega_{3}+\omega_{4}+\omega_{3}^{\prime}+\omega_{4}^{\prime}, 0}+\delta_{\omega_{3}+\omega_{4}+\omega_{3}^{\prime}+\omega_{4}^{\prime}, 0} \delta_{\omega_{1}+\omega_{2}+\omega_{3}^{\prime}+\omega_{4}^{\prime}, 0}\right]
\end{aligned}
$$

and hence $\gamma^{[2]}=\frac{g}{4} L$. Since we have kept only the contribution of $\mathcal{O}(g)$ to the reduced $2 \mathrm{PI} 4$-point vertex function $\bar{\gamma}^{[2]}$, the expansion (54) of the functional $\bar{\Gamma}^{\mathrm{E} 2 \mathrm{PC}}[G]$ in powers of the free 2-particle propagator $\mathcal{G}^{\text {tree }} \sim(G G)^{I}$ corresponds to its expansion in powers of $g$. The $2 \mathrm{PI}$ effective action takes now the form

$$
\begin{aligned}
\Gamma^{\mathrm{E} 2 \mathrm{PC}}[G]= & \Gamma^{\mathrm{tree}}[G]+\frac{g}{8} \int_{\omega_{1}, \ldots, \omega_{4}} \delta_{\omega_{1}+\omega_{2}+\omega_{3}+\omega_{4}, 0} G_{\omega_{1}, \omega_{2}} G_{\omega_{3}, \omega_{4}} \\
& -\frac{i g^{2}}{48 \hbar} \int_{\omega_{1}, \ldots, \omega_{4}, \omega_{1}^{\prime} \ldots, \omega_{4}^{\prime}} \delta_{\omega_{1}+\omega_{2}+\omega_{1}^{\prime}+\omega_{2}^{\prime}, 0} \delta_{\omega_{3}+\omega_{4}+\omega_{3}^{\prime}+\omega_{4}^{\prime}, 0} G_{\omega_{1}, \omega_{3}} G_{\omega_{2}, \omega_{4}} G_{\omega_{1}^{\prime}, \omega_{3}^{\prime}} G_{\omega_{2}^{\prime}, \omega_{4}^{\prime}}+\mathcal{O}\left(G^{6}\right) .
\end{aligned}
$$

In this approximation based on the E2PC there occurs an additional contribution of the order $\mathcal{O}\left(g^{2}\right)$ in the $2 \mathrm{PI}$ effective action which was not included in the IMA: in the right-hand side of Eq. (61) there occurs an additional factor 3 in the term of the order $\mathcal{O}\left(g^{2}\right)$ as compared to the corresponding term in the IMA. This is because of the improvement of the 2-particle propagator,

$$
\mathcal{G}^{\mathrm{E} 2 \mathrm{PC}}[G]=\mathcal{G}^{\text {tree }}[G]+\frac{g}{4} \mathcal{G}^{\text {tree }}[G]: L: \mathcal{G}^{\text {tree }}[G]+\mathcal{O}\left(g^{2}\right)
$$

(c.f. Eq. (57)) as compared to the free one used in the IMA. Putting it in another way, in the approximation base on the E2PC we use the 2PI 4-point vertex function $\gamma^{[2]}=\bar{\gamma}^{[2]}+\gamma^{[2] \text { tree }}=(g / 4) L$ instead of $\gamma^{[2] \text { tree }}=(g / 12) L$ used in the IMA. The diagrammatic form of RG the evolution equation remains the same as that in the IMA (see Fig. 10) except of replacing the vertices $\gamma^{[2]}$ tree by $\gamma^{[2]}$ of the present approximation.

The necessary condition of the extremum of the $2 \mathrm{PI}$ effective action, $\delta \Gamma^{\mathrm{E} 2 \mathrm{PC}}[G] / \delta G=0$ provides again the Schwinger-Dyson equation for the propagator of the ground state,

$$
\begin{aligned}
G_{\mathrm{gr} \omega_{1}, \omega_{2}}^{\mathrm{E} 2 \mathrm{PC}-1}= & G_{\mathrm{gr} \omega_{1}, \omega_{2}}^{\mathrm{tree}-1}+\left[\frac{i g}{2 \hbar} \int_{\omega_{1}^{\prime}, \omega_{2}^{\prime}} \delta_{\omega_{1}+\omega_{2}+\omega_{1}^{\prime}+\omega_{2}^{\prime}, 0} G_{\omega_{1}^{\prime}, \omega_{2}^{\prime}}\right. \\
& \left.+\frac{g^{2}}{6 \hbar^{2}} \int_{\omega_{1}^{\prime}, \ldots, \omega_{4}^{\prime}, \omega_{3}, \omega_{4}} \delta_{\omega_{1}+\omega_{3}+\omega_{1}^{\prime}+\omega_{2}^{\prime}, 0} \delta_{\omega_{2}+\omega_{4}+\omega_{3}^{\prime}+\omega_{4}^{\prime}, 0} G_{\omega_{3}, \omega_{4}} G_{\omega_{1}^{\prime}, \omega_{3}^{\prime}} G_{\omega_{2}^{\prime}, \omega_{4}^{\prime}}\right]_{G=G_{\mathrm{gr}}^{\mathrm{E} 2 \mathrm{PC}}}
\end{aligned}
$$

and a straightforward but lengthy calculation yields

$$
\begin{aligned}
& \gamma_{\left(\omega_{3}, \omega_{4}\right),\left(\omega_{1}, \omega_{2}\right)}^{[2] \mathrm{E} 2 \mathrm{PC}}=\mathcal{G}_{\left(\omega_{3}, \omega_{4}\right),\left(\omega_{1}, \omega_{2}\right)}^{\mathrm{E} 2 \mathrm{PC}-1 I}\left[G_{\mathrm{gr}}^{\mathrm{E} 2 \mathrm{PC}}\right]-\mathcal{G}_{\left(\omega_{3}, \omega_{4}\right),\left(\omega_{1}, \omega_{2}\right)}^{\mathrm{tree}-1 I}\left[G_{\mathrm{gr}}^{\mathrm{E} 2 \mathrm{PC}}\right] \\
& \quad=\frac{g}{4} \delta_{\omega_{1}+\omega_{2}+\omega_{3}+\omega_{4}, 0}-\frac{i g^{2}}{8 \hbar} \int_{\omega_{1}^{\prime}, \omega_{2}^{\prime}, \omega_{3}^{\prime}, \omega_{4}^{\prime}}\left[\delta_{\omega_{1}+\omega_{3}+\omega_{1}^{\prime}+\omega_{3}^{\prime}, 0} \delta_{\omega_{2}+\omega_{4}+\omega_{2}^{\prime}+\omega_{4}^{\prime}, 0}+\left(\omega_{1} \Leftrightarrow \omega_{2}\right)\right]\left[G_{\omega_{1}^{\prime}, \omega_{2}^{\prime}} G_{\omega_{3}^{\prime}, \omega_{4}^{\prime}}\right]_{G=G_{\mathrm{gr}} \mathrm{EC}}
\end{aligned}
$$

for the value of the 2PI 4-point vertex in the ground state. The $\mathcal{O}\left(g^{2}\right)$ term of the propagator as well as that of the 2PI vertex function exhibit the additional factor 3 as compared to the corresponding terms in the IMA.

\section{Determination of the renormalized couplings}

We shall obtain the renormalized quantities in the IS RG scheme in the IMA and in the truncated E2PC, as described previously in Sections III B 1 and III B 2, respectively, in both cases keeping the terms up to the second 
order $\mathcal{O}\left(g^{2}\right)$ of the bare coupling $g$ that has been used as the control parameter. We shall make use of the diagonal form of the propagator, $G_{\omega, \omega^{\prime}}=G_{\omega} \delta_{\omega, \omega^{\prime}}$. First, we determine the propagator $G_{\mathrm{gr} \omega}$ of the ground state by solving the Schwinger-Dyson equation with the precision $\mathcal{O}\left(g^{2}\right)$, and afterwards the energy of the ground state via

$$
E_{0}=\lim _{T \rightarrow \infty} \frac{1}{T} \Gamma\left[G_{\mathrm{gr}}\right]
$$

and the renormalized coupling $g_{0}$ from the 2PI 4-point vertex function taken at the symmetric point $\omega_{i}=0(i=$ $1,2,3,4)$ via

$$
g_{0}=\lim _{T \rightarrow \infty, \omega_{i} \rightarrow 0} \frac{4}{T} \gamma_{\left(\omega_{3}, \omega_{4}\right),\left(\omega_{1}, \omega_{2}\right)}^{[2]} .
$$

Finally, we compare these results with those obtained at the same order of the perturbation expansion in the CS RG scheme given in Appendix A 3, in the WH RG scheme given in Appendix B 2 b, as well as by the results of the well-known Rayleigh-Schrödinger perturbation expansion in quantum mechanics.

First, let us present the results obtained in the framework of the IS RG in the IMA. For diagonal propagator the Schwinger-Dyson equation (51) can be rewritten as

$$
G_{\mathrm{gr} \omega}^{\mathrm{IMA}-1}=G_{\mathrm{gr} \omega}^{\mathrm{tree}}-1+\left[\frac{i g}{2 \hbar} \int_{\omega_{1}} G_{\omega_{1}}+\frac{g^{2}}{18 \hbar^{2}} \int_{\omega_{1}, \omega_{2}, \omega_{3}} \delta_{\omega+\omega_{1}+\omega_{2}+\omega_{3}, 0} G_{\omega_{1}} G_{\omega_{2}} G_{\omega_{3}}+\mathcal{O}\left(g^{3}\right)\right]_{G=G_{\mathrm{gr}}^{\mathrm{IMA}}} .
$$

Let us insert into Eq. (67) the perturbation expansion

$$
G^{-1}=G_{0}^{-1}+g A+g^{2} B+\mathcal{O}\left(g^{3}\right), \quad G=G_{0}-g G_{0} \cdot A \cdot G_{0}-g^{2} G_{0} \cdot C \cdot G_{0}+\mathcal{O}\left(g^{3}\right)
$$

where $A$ and $B$ are symmetric matrices to be determined, $C=B-A \cdot G_{0} \cdot A$ and we wrote $G_{0}=G_{\mathrm{gr}}^{\text {tree }}$ for the sake of simplicity. Comparing the terms of identical powers of the coupling $g$ in both sides of Eq. (67), one finds $A_{\omega, \omega^{\prime}}=A_{\omega} \delta_{\omega+\omega^{\prime}, 0}$ and $B_{\omega, \omega^{\prime}}=B_{\omega} \delta_{\omega+\omega^{\prime}, 0}$ with

$$
A_{\omega}=\frac{i}{2 \hbar} \int_{\omega_{1}} G_{0 \omega_{1}}, \quad B_{\omega}=\frac{1}{4 \hbar^{2}} \int_{\omega_{1}} G_{0 \omega_{1}}^{2} \int_{\omega_{2}} G_{0 \omega_{2}}+\frac{1}{18 \hbar^{2}} \int_{\omega_{1}, \omega_{2}} G_{0 \omega_{1}+\omega_{2}-\omega} G_{0 \omega_{1}} G_{0 \omega_{2}} .
$$

The loop integrals can be taken explicitly making use of the residuum theorem,

$$
\int_{\omega} G_{0} \omega=\frac{\hbar}{2 \omega_{0}}, \quad \int_{\omega} G_{0}^{2} \omega=\frac{-i \hbar^{2}}{4 \omega_{0}^{3}}, \quad \int_{\omega_{1}, \omega_{2}} G_{0} \omega_{1}+\omega_{2}-\omega G_{0 \omega_{1}} G_{0} \omega_{2}=\frac{3 i \hbar^{3}}{4 \omega_{0}^{2}} \frac{1}{\omega^{2}-9 \omega_{0}^{2}+18 i \epsilon} .
$$

Expanding the last loop integral in powers of $\omega^{2}-\omega_{0}^{2}$ and keeping the terms up to the order $\mathcal{O}\left(\omega^{2}-\omega_{0}^{2}\right)$, one finds for the inverse of the propagator of the ground state

$$
G_{\mathrm{gr} \omega}^{\mathrm{IMA}-1}=-\frac{i}{\hbar}\left(x_{0} \omega^{2}-\Omega_{0}^{2}+i \epsilon\right)
$$

with

$$
\Omega_{0}^{2}=\omega_{0}^{2}\left[1+4 \xi-\frac{55}{6} \xi^{2}\right]+\mathcal{O}\left(g^{3}\right), \quad x_{0}=1+\frac{1}{3} \xi^{2}+\mathcal{O}\left(g^{3}\right)
$$

in terms of the dimensionless parameter $\xi=\frac{g \hbar}{16 \omega_{0}^{3}}$, introduced previously. For diagonal propagator $G$ the $2 \mathrm{PI}$ effective action given by Eqs. (40) and (49) and the 2PI 4-point vertex function (53) reduce to

$$
\Gamma^{\mathrm{IMA}}[G]=\bar{\Gamma}^{\text {tree }}[G]+\frac{g}{8} T\left(\int_{\omega} G_{\omega}\right)^{2}-\frac{i g^{2}}{144 \hbar} T \int_{\omega_{1}, \omega_{2}, \omega_{3}} G_{\omega_{1}} G_{\omega_{2}} G_{\omega_{3}} G_{\omega_{1}+\omega_{2}+\omega_{3}}+\mathcal{O}\left(G^{6}\right)
$$

and

$$
\gamma_{\left(\omega_{3}, \omega_{4}\right),\left(\omega_{1}, \omega_{2}\right)}^{[2] I M A}=\left[\frac{g}{4}-\frac{i g^{2}}{24 \hbar} \int_{\omega} G_{\omega}\left(G_{-\omega_{1}-\omega_{3}-\omega}+G_{-\omega_{2}-\omega_{3}-\omega}\right)\right] \delta_{\omega_{1}+\omega_{2}+\omega_{3}+\omega_{4}, 0},
$$


respectively. Inserting $G=G_{\mathrm{gr}}^{\mathrm{IMA}}$ into the expression (73) one finds for the energy of the ground state

$$
E_{0}=\frac{1}{2} \hbar \omega_{0}\left[1+\xi-\frac{55}{9} \xi^{2}+\mathcal{O}\left(g^{3}\right)\right]
$$

According to Eqs. (66) and (74), one obtains for the renormalized quartic coupling,

$$
g_{0}=g-\frac{i g^{2}}{3 \hbar} \int_{\omega} G_{0 \omega}^{2}+\mathcal{O}\left(g^{3}\right)=g\left[1-\frac{4}{3} \xi+\mathcal{O}\left(g^{2}\right)\right] .
$$

At second, we turn to the approximation based on the E2PC. Making use of Eqs. (61), (63), and (64), one can proceed like in the case of the IMA and obtain results with slightly different factors for the second-order terms,

$$
\begin{gathered}
\Omega_{0}^{2}=\omega_{0}^{2}\left[1+4 \xi-\frac{71}{8} \xi^{2}+\mathcal{O}\left(\xi^{3}\right)\right], \quad x_{0}=1+\frac{1}{8} \xi^{2}+\mathcal{O}\left(\xi^{3}\right), \\
E_{0}=\frac{1}{2} \hbar \omega_{0}\left[1+\xi-\frac{16}{3} \xi^{2}+\mathcal{O}\left(\xi^{3}\right)\right] \\
g_{0}=g\left(1-4 \xi+\mathcal{O}\left(\xi^{2}\right)\right) .
\end{gathered}
$$

Finally, in Table I we compare the results obtained in the various approximation schemes. The various observables are given keeping the terms up to the order $\mathcal{O}\left(g_{B}^{2}\right)$ of the bare coupling. We compare here the renormalized values, ie. the IR limits of them obtained in the CSi RG scheme in second order of the perturbation expansion as given in Appendix A 3, the renormalized values obtained in the ISg RG scheme in IMA and in the approximation based on the E2PC when the evolution has been ended up and the control parameter reached the same bare value $g_{B}$ that used in the CSi RG scheme. We also present the results obtained in the framework of the WH RG scheme as outlined in Appendix B 2 b, and the results of the Rayleigh-Schrödinger perturbation expansion (RSPE) [39]. In the RSPE one finds for the energy of the $n$-th energy niveau $(n=0,1,2, \ldots)$ of the one-dimensional anharmonic oscillator with the bare Hamiltonian $H=\frac{1}{2} p^{2}+\frac{1}{2} \omega_{0}^{2} x^{2}+\frac{g_{B}}{24} x^{4}$,

$$
E_{n}=\hbar \omega_{0}\left[n+\frac{1}{2}+e_{n}^{(1)}+e_{n}^{(2)}+\mathcal{O}\left(\xi^{3}\right)\right]
$$

with

$$
\begin{aligned}
e_{n}^{(1)}= & \left(1+2 n+2 n^{2}\right) \xi \\
e_{n}^{(2)}= & -\frac{1}{18} \xi^{2} \sum_{n^{\prime} \neq n} \frac{1}{n^{\prime}-n}\left[4(2 n-1)^{2} n(n-1) \delta_{n^{\prime}, n-2}+n(n-1)(n-2)(n-3) \delta_{n^{\prime}, n-4}\right. \\
& \left.+4(2 n+3)^{2}(n+1)(n+2) \delta_{n^{\prime}, n+2}+(n+1)(n+2)(n+3)(n+4) \delta_{n^{\prime}, n+4}\right] .
\end{aligned}
$$

This yields for the first two energy levels

$$
\begin{aligned}
& E_{0}=\frac{1}{2} \hbar \omega_{0}\left(1+\xi-\frac{7}{3} \xi^{2}+\mathcal{O}\left(\xi^{3}\right)\right) \\
& E_{1}=\frac{1}{2} \hbar \omega_{0}\left(3+5 \xi-\frac{55}{3} \xi^{2}+\mathcal{O}\left(\xi^{3}\right)\right)
\end{aligned}
$$

and

$$
\left(\hbar \Omega_{0}\right)^{2}=\left(E_{1}-E_{0}\right)^{2}=\left(\hbar \omega_{0}\right)^{2}\left(1+4 \xi-12 \xi^{2}\right) .
$$

The same expression for the energy $E_{0}$ of the ground-state has been found in Ref. [37] by path-integral method. There are no asymptotically free states of the particle moving in the quartic anharmonic oscillator potential, therefore the definition of scattering amplitudes and through those a renormalized quartic coupling is problematic in the RSPE approach.

All the RG schemes reproduce the first-order corrections in accord with the RSPE. This is because these corrections are one-loop corrections according to the loop expansion and the essentially one-loop RG equations describe the quantum effects at the one-loop order exactly, independently of the approximation schemes additionally used. We see that all the RG schemes have it common that the second-order corrections deepen the energy levels, tend to decrease the quartic coupling and result in a field-independent wavefunction renormalization $x_{0} \geq 1$. Nevertheless, the truncation of the perturbation expansion causes a strong dependence of the quantitative results on the RG scheme used. 


\begin{tabular}{|c|c|c|c|c|c|}
\hline Observable & ISg RG IMA & ISg RG E2PC & CSi RG & WH RG & RSPE \\
\hline$E_{0}-\frac{1}{2} \hbar \omega_{0}-\xi$ & $-\frac{55}{9} \xi^{2}$ & $-\frac{16}{3} \xi^{2}$ & $-\frac{71}{30} \xi^{2}$ & $-18 \xi^{2}$ & $-\frac{7}{3} \xi^{2}$ \\
\hline$\frac{\Omega_{0}^{2}-\omega_{0}^{2}}{\omega_{0}^{2}}-4 \xi$ & $-\frac{55}{6} \xi^{2}$ & $-\frac{71}{8} \xi^{2}$ & $-11 \xi^{2}$ & $-32 \xi^{2}$ & $-12 \xi^{2}$ \\
\hline$\frac{g_{0}-g_{B}}{g_{B}}$ & $-\frac{4}{3} \xi$ & $-4 \xi$ & $-6 \xi$ & $-6 \xi$ & 0 \\
\hline$x_{0}-1$ & $\frac{1}{3} \xi^{2}$ & $\frac{1}{8} \xi^{2}$ & $\frac{2}{3} \xi^{2}$ & 0 & 0 \\
\hline
\end{tabular}

TABLE I: Comparison of the observables obtained in the framework of various RG schemes. The expansion in the dimensionless parameter $\xi=\frac{g_{B} \hbar}{16 \omega_{0}^{3}}$ is truncated keeping the terms up to the second order.

\section{CONCLUSIONS}

The role of wavefunction renormalization and dependence of the observables on the RG scheme in the second order perturbation expansion has been investigated on the example of the anharmonic oscillator. The functional CSi RG scheme with the imaginary mass and the ISg RG with the bare quartic coupling as control parameters have been applied to the 1PI and 2PI effective actions, respectively. The CSi RG evolution equation derived for the $N$-dimensional $O(N)$ symmetric oscillator has been solved by making a quartic Ansatz for the 1PI effective action including terms for field-dependent wavefunction renormalization, since that is the only way to generate field-independent wavefunction renormalization by means of a one-loop evolution equation. The evolution equation has been solved keeping the terms of the Neumann-expansion of the inverse matrix in the right-hand side of the evolution equation only up to the second order in the renormalized propagator and those up to the quartic ones in the oscillator coordinate. It has been shown that the $O(N)$ symmetric anharmonic oscillator exhibits only a single phase, independently of its dimension $N$. Furthermore, the inclusion of next-to-leading order of the gradient expansion, the wavefunction renormalization does not modify qualitatively neither the IR scaling laws nor the phase structure. It has also been established that the effect of wavefunction renormalization decreases with increasing dimension $N$ of the oscillator according to a power-law.

The ISg RG has been applied to the 2PI effective action, in order to obtain a one-looplike RG evolution equation again. Our study was restricted to the one-dimensional oscillator in that case. An approximation scheme based on the E2PC, going beyond the IMA, has been put forward that corresponds the expansion of the 2PI effective action into the powers of the free 2-particle propagator $\mathcal{G}^{\text {tree }}$. The RG evolution equation for the $2 \mathrm{PI}$ effective action has been rewritten as a coupled set of evolution equations for the 2PI 4-point and 8-point vertex functions by keeping the terms up to those of the order $\mathcal{O}\left(\left(\mathcal{G}^{\text {tree }}\right)^{4}\right)$ in the effective action and those of the order $\mathcal{O}\left(\left(\mathcal{G}^{\text {tree }}\right)^{2}\right)$ in the Neumann-expansion of the inverse of the full-particle propagator. The solutions for the vertex functions have been inserted into the Schwinger-Dyson equation for the propagator of the ground state that has been solved by making an Ansatz for the latter assuming the dominance of a single pole. The advantages of that ISg RG method as compared to the CSi RG scheme are that it enables one to read off the field-independent wavefunction renormalization directly and it provides an UV finite result for the ground-state energy.

Finally we compared the results obtained for the ground-state energy, the 'mass' (in field-theoric term), the renormalized quartic coupling, and the field-independent wavefunction renormalization in the various RG schemes (ISg RG, CSi RG, WH RG) and the Rayleigh-Schrödinger perturbation expansion (RSPE) restricting ourselves to the second-order perturbation expansion in powers of the bare quartic coupling $g_{B}$. While the first-order contributions and the sign of the second-order ones are reproduced by all of these approximations there has been found a remarkable scheme-dependence of the weights of the second-order terms. 


\section{Acknowledgements}

The authors thank Janos Polonyi for useful discussions. Our work is supported by the project TÁMOP 4.2.1-08/12008-003. The project is implemented through the New Hungary Development Plan co-financed by the European Social Fund, and the European Regional Development Fund.

[1] R. Hedden, V. Meden, Th. Pruschke, K. Schönhammer, J. Phys.: Cond.Matter, 16, 5279 (2004).

[2] S. Huang, G.S. Agarwal, Phys. Rev. A80, 033807 (2009).

[3] V. Barsan, 0912.1988 [cond-mat.stat-mech].

[4] D.J. Scalapino, M. Sears, R.A. Ferrell, Phys. Rev. B6, 3409 (1972).

[5] J.A. Krumhansl, J.R. Schrieffer, Phys. Rev. B11, 3535 (1975).

[6] A. Kowalewska-Kudłaszyk, J.K.Kalaga, W.Leoński, Phys. Rev. E 78, 066219 (2008); ibid. Phys. Lett. A373, 1334 (2009).

[7] T.V.Gevorgyan, A.R.Shahinyan, G.Yu. Kryuchkyan, Phys. Rev. A79, 053828 (2009).

[8] Ch. Skokos, D.O.Krimer, S. Komineas, S. Flach, Phys. Rev. E79, 056211 (2009).

[9] A. Kapulkin, A. K. Pattanayak, Phys. Rev. Lett. 101, 074101 (2008); J.Finn, K. Jacobs, B.Sundaram, Phys. Rev. Lett. 102, 119401 (2009); K. Kingsbury, C. Amey, A. Kapulkin, A. Pattanayak, Phys. Rev. Lett. 102, 119402 (2009).

[10] C.M. Bender, T.T. Wu, Phys.Rev. 184, 1231 (1969).

[11] C.M. Bender, J.E. Mandula, B.M. McCoy, Phys. Rev. Lett. 24, 681 (1970); C.M. Bender, T.T. Wu, Phys. Rev. Lett. 27, 461 (1971); ibid. Phys. Rev. D7, 1620 (1973).

[12] C.M. Bender, F. Cooper, G.S. Guralnik, D.H. Sharp, Phys. Rev. D19, 1865 (1979).

[13] C.M. Bender, G.V. Dunne, Phys. Rev. D40, 2739 (1989); ibid. Phys. Rev. D40, 3504 (1989).

[14] C.M. Bender, L.M.A. Bettencourt, Phys. Rev. Lett. 77, 4114 (1996); ibid. Phys. Rev. D54, 7710 (1996); G. Auberson, M. C. Peyranre, Phys. Rev. A65, 032120 (2002).

[15] K. Vlachos, Phys. Rev. A47, 838 (1993); Y. Zhou, J.D. Mancini, P.F. Meier, S.P. Bowen, Phys. Rev. A51, 3337 (1995); V. Fessatidis, J.D. Mancini, J.D. Prie, Y. Zhou, A. Majewski, Phys. Rev. A60, 1713 (1999).

[16] R. Jáuregui, J. Récamier, Phys. Rev. A46, 2240 (1992).

[17] C.R. Handy,Phys. Rev. A46, 1663 (1992).

[18] R.N. Chaudhuri, M. Mondal,Phys. Rev. A43, 3241 (1991); R.K. Agrawal, V.S. Varma, Phys. Rev. A49, 5089 (1994).

[19] T. Hatsuda, T. Kunihiro, T. Tanaka, Phys. Rev. Lett. 78, 3229 (1997).

[20] H. Meißner, E.O. Steinborn, Phys. Rev. A56, 1189 (1997).

[21] T. Kashiwa, Phys. Rev. D 59, 085002 (1999).

[22] Sangchul Oh, Phys. Rev. A77, 012326 (2008).

[23] T. Kunihiro, Phys. Rev. D57, R2035 (1998); I.L. Egusquiza, M.A.V. Basagoiti, Phys. Rev. A57, 1586 (1998).

[24] V. I. Yukalov, E. P. Yukalova, and S. Gluzman, Phys. Rev. A58, 96 (1998).

[25] M. Frasca, Nuovo Cim. B117, 867 (2002).

[26] A.S. Kapoyannis, N. Tetradis, Phys. Lett. A276, 225 (2000).

[27] K-I Aoki, A. Horikoshi, M. Taniguchi, H. Terao, Prog. Theor. Phys., 108, 571 (2002).

[28] K.G. Wilson, J. Kogut,Phys. Rep. C12, 77 (1974); K.G. Wilson, Rev. Mod. Phys. 47, 773 (1975); ibid. Rev. Mod. Phys. 55, 583 (1983).

[29] F.J. Wegner, A. Houghton, Phys. Rev. A8, 401 (1973).

[30] J. Polchinski, Nucl. Phys. B231, 269 (1984).

[31] C. Wetterich, Phys. Lett. B301, 90 (1993).

[32] T. Morris, Int. J. Mod. Phys. A9, 2411 (1994).

[33] C.G. Callan, Phys. Rev. D2, 1541 (1970); K. Symanzik, Comm. Math. Phys. 18, 227 (1970).

[34] M. Simionato, Int. J. Mod. Phys. A15, 2121 (2000); J. Alexandre, J. Polonyi, Ann. Phys. 288, 37 (2001).

[35] J. Polonyi, K. Sailer, Phys. Rev. D71, 025010 (2005).

[36] C.De Dominicis, P.C. Martin, J. Math. Phys. 5, 14 (1964); J.M. Cornwall, R. Jackiw, E. Tomboulis, Phys. Rev. D10, 2428 (1974).

[37] R.W. Haymaker, Nuovo Cim., 14, 1 (1991).

[38] A. Margaritis, G. Ódor, A. Patkos, Z. Phys. C39, 109 (1998).

[39] L.D. Landau, E.M. Lifshitz, Quantum Mechanics. Nonrelativistic Theory, Pergamon Press, Oxford (1965).

[40] W. Thomas, Naturwissenschaften13, 627 (1925); F. Reiche, W. Thomas, Z. Phys. 34, 510 (1925); W. Kuhn, Z. Phys. 33, 408 (1925).

[41] J. Polonyi, Central Eur. J. Phys. 1, 1 (2004); S. Nagy, I. Nándori, J. Polonyi, K. Sailer, Phys. Lett B647, 152 (2007); S. Nagy, I. Nándori, J. Polonyi, K. Sailer, Phys. Rev. D77, 025026 (2008).

[42] R. Feynman, Statistical Mechanics, chapt. 3, (W.A.Benjamin, Inc., Massachusetts, 1972). 


\section{Appendix A: CSi RG scheme for the $N$-dimensional anharmonic oscillator in real-time formalism}

\section{Neumann-expansion}

For later use let us introduce the matrices

$$
C_{\omega, \omega^{\prime}}=\bar{C}_{(c, \omega),\left(c, \omega^{\prime}\right)}=C_{\omega^{\prime}, \omega}
$$

and

$$
\begin{aligned}
& B_{\omega,-\omega}=-2 \int_{\omega_{1}}\left[v\left(\omega_{1},-\omega_{1}\right)+v(\omega,-\omega)\right] q_{a, \omega_{1}} q_{a,-\omega_{1}}=-2 \int_{\omega_{1}}\left(-\frac{1}{2} y\left(\omega_{1}^{2}+\omega^{2}\right)+\frac{g}{12}\right) q_{a, \omega_{1}} q_{a,-\omega_{1}}, \\
& C_{\omega,-\omega}=-4 \int_{\omega_{1}}\left[v\left(\omega_{1},-\omega\right)+v\left(-\omega_{1}, \omega\right)\right] q_{a, \omega_{1}} q_{a,-\omega_{1}}=-8 \int_{\omega_{1}}\left(-\frac{1}{2} \bar{y} \omega_{1} \omega+\frac{1}{4} \bar{Y}\left(\omega_{1}^{2}+\omega^{2}\right)+\frac{g}{24}\right) q_{a, \omega_{1}} q_{a,-\omega_{1}} .
\end{aligned}
$$

The first two terms in the expansion (14) are quadratic in the field variable $q_{a, \omega}$ and can be expressed in terms of the loop integrals $I_{n, s}$ defined in Eq. (17) in a straightforward manner,

$$
\begin{aligned}
&-\frac{N \hbar}{2} \int_{\omega} G_{\omega}=-\frac{N}{2} I_{1,0}, \\
& \frac{\hbar}{2} \int_{\omega} G_{\omega}^{2} A_{(a, \omega),(a,-\omega)}= \frac{N \hbar}{2} \int_{\omega} G_{\omega}^{2} B_{\omega,-\omega}+\frac{\hbar}{2} \int_{\omega} G_{\omega}^{2} C_{\omega,-\omega} \\
&= \frac{1}{2}[N \bar{y}-(N+2) \bar{Y}] I_{2,0} \int_{\omega_{1}} \omega_{1}^{2} q_{a, \omega_{1}} q_{a,-\omega_{1}} \\
&+\frac{1}{2}\left([N \bar{y}-(N+2) \bar{Y}] I_{2,2}-\frac{(N+2) g}{6} I_{2,0}\right) \int_{\omega_{1}} q_{a, \omega_{1}} q_{a,-\omega_{1}} .
\end{aligned}
$$

The third term of the series (14) can be rewritten as

$$
\begin{aligned}
& -\frac{\hbar}{2} \int_{\omega, \omega^{\prime}} G_{\omega}^{2} A_{(c, \omega),\left(d, \omega^{\prime}\right)} G_{\omega^{\prime}} A_{\left(d,-\omega^{\prime}\right),(c,-\omega)} \\
& =-\frac{\hbar}{2} \int_{\omega, \omega^{\prime}} G_{\omega}^{2} G_{\omega^{\prime}}\left[N B_{\omega, \omega^{\prime}} B_{-\omega,-\omega^{\prime}}+2 B_{-\omega,-\omega^{\prime}} C_{\omega, \omega^{\prime}}+\bar{C}_{(c, \omega),\left(d, \omega^{\prime}\right)} \bar{C}_{(c,-\omega),\left(d,-\omega^{\prime}\right)}\right] \\
& =T_{1}+T_{2}+T_{3},
\end{aligned}
$$

where all terms are quartic in the Fourier-transform of the field variable,

$$
\begin{aligned}
T_{1}= & -\frac{N \hbar}{2} \int_{\omega, \omega^{\prime}} G_{\omega}^{2} G_{\omega^{\prime}} B_{\omega, \omega^{\prime}} B_{-\omega,-\omega^{\prime}} \\
= & -2 N \hbar \int_{\omega, \omega^{\prime}} G_{\omega}^{2} G_{\omega^{\prime}} \int_{\omega_{1}, \ldots, \omega_{4}} \delta_{\omega_{1}+\omega_{2}+\omega_{3}+\omega_{4}, 0} \delta_{\omega+\omega^{\prime}+\omega_{1}+\omega_{2}, 0}\left(v\left(\omega_{1}, \omega_{2}\right) v\left(\omega_{3}, \omega_{4}\right)\right. \\
& \left.+2 v\left(\omega, \omega^{\prime}\right) v\left(\omega_{1}, \omega_{2}\right)+v^{2}\left(\omega, \omega^{\prime}\right)\right) q_{a, \omega_{1}} q_{a, \omega_{2}} q_{b, \omega_{3}} q_{b, \omega_{4}}, \\
T_{2}=- & \hbar \int_{\omega, \omega^{\prime}} G_{\omega}^{2} G_{\omega^{\prime}} B_{-\omega,-\omega^{\prime}} C_{\omega, \omega^{\prime}} \\
= & -8 \hbar \int_{\omega, \omega^{\prime}, \omega_{1}, \omega_{2}, \omega_{3}, \omega_{4}} G_{\omega}^{2} G_{\omega^{\prime}} \delta_{\omega_{1}+\omega_{2}+\omega_{3}+\omega_{4}, 0} \delta_{-\omega-\omega^{\prime}+\omega_{1}+\omega_{2}, 0}\left(v\left(\omega_{1}, \omega_{2}\right)\left[v\left(\omega_{3}, \omega\right)+v\left(\omega_{3}, \omega^{\prime}\right)\right]\right. \\
& \left.+v\left(\omega, \omega^{\prime}\right)\left[v\left(\omega_{3}, \omega\right)+v\left(\omega_{3}, \omega^{\prime}\right)\right]\right) q_{a, \omega_{1}} q_{a, \omega_{2}} q_{b, \omega_{3}} q_{b, \omega_{4}},
\end{aligned}
$$




$$
\begin{aligned}
T_{3}= & -\frac{\hbar}{2} \int_{\omega, \omega^{\prime}} G_{\omega}^{2} G_{\omega^{\prime}} \bar{C}_{(c, \omega),\left(d, \omega^{\prime}\right)} \bar{C}_{(c,-\omega),\left(d,-\omega^{\prime}\right)} \\
= & -8 \hbar \int_{\omega_{1}, \ldots, \omega_{4}, \omega, \omega^{\prime}} G_{\omega}^{2} G_{\omega^{\prime}} \delta_{\omega_{1}+\ldots+\omega_{4}, 0} \delta_{\omega_{1}+\omega_{3}+\omega+\omega^{\prime}, 0} \\
& \times\left[v\left(\omega_{1}, \omega\right) v\left(\omega_{2},-\omega\right)+v\left(\omega_{1}, \omega\right) v\left(\omega_{4},-\omega^{\prime}\right)+v\left(\omega_{3}, \omega^{\prime}\right) v\left(\omega_{2},-\omega\right)+v\left(\omega_{3}, \omega^{\prime}\right) v\left(\omega_{4},-\omega^{\prime}\right)\right] \\
& \times q_{a, \omega_{1}} q_{a, \omega_{2}} q_{b, \omega_{3}} q_{b, \omega_{4}} .
\end{aligned}
$$

Evaluating $T_{1}, T_{2}$, and $T_{3}$ we keep only the terms corresponding to the interaction vertices included in the Ansatz (6) for the effective action in the truncated gradient expansion, ie. we keep self-interaction terms up to the quartic ones and quadratic gradient terms with wave-function renormalization truncated at the quadratic field-dependent term. By making use of the Dirac-deltas expressing energy conservation in the vertices, $G_{\omega^{\prime}}$ can be changed to $G_{\omega+\alpha}$ with $\alpha= \pm\left(\omega_{1}+\omega_{2}\right)$. In order to reveal the terms of the gradient expansion explicitly, we have to expand the propagators with shifted frequency $G_{\omega+\alpha}$ in the Taylor series (16) at $\alpha=0$. Furthermore, we also make use of the identity (9), in order to transform all gradient terms in one of those of the Ansatz (6). A rather lengthy but straightforward calculation yields finally,

$$
\begin{aligned}
& T_{1}=-2 N \int_{\omega_{1}, \ldots, \omega_{4}} \delta_{\omega_{1}+\omega_{2}+\omega_{3}+\omega_{4}, 0}\left\{\frac{1}{4} y^{2} I_{3,4}-2 \frac{g}{24} y I_{3,2}+4 \frac{g^{2}}{24^{2}} I_{3,0}\right. \\
& +\left[2 y^{2} x^{2} I_{5,6}-16 \frac{g}{24} y x^{2} I_{5,4}+32 \frac{g^{2}}{24^{2}} x^{2} I_{5,2}\right. \\
& -\frac{5}{2} x y^{2} I_{4,4}+12 \frac{g}{24} x y I_{4,2}-8 \frac{g^{2}}{24^{2}} x I_{4,0} \\
& \left.+\frac{1}{2} \bar{y}^{2} I_{3,2}-2 \bar{y} \bar{Y} I_{3,2}+\frac{3}{2} \bar{Y}^{2} I_{3,2}+4 \frac{g}{24} \bar{Y} I_{3,0}\right] \omega_{1}^{2} \\
& +\left[2 y^{2} x^{2} I_{5,6}-16 \frac{g}{24} y x^{2} I_{5,4}+32 \frac{g^{2}}{24^{2}} x^{2} I_{5,2}\right. \\
& -\frac{5}{2} x y^{2} I_{4,4}+12 \frac{g}{24} y x I_{4,2}-8 \frac{g^{2}}{24^{2}} x I_{4,0} \\
& \left.\left.-\bar{y} \bar{Y} I_{3,2}+\bar{Y}^{2} I_{3,2}+2 \frac{g}{24} \bar{y} I_{3,0}+2 \frac{g}{24} \bar{Y} I_{3,0}\right] \omega_{1} \omega_{2}\right\} q_{a, \omega_{1}} q_{a, \omega_{2}} q_{b, \omega_{3}} q_{b, \omega_{4}}, \\
& T_{2}=-8 \int_{\omega_{1}, \omega_{2}, \omega_{3}, \omega_{4}} \delta_{\omega_{1}+\omega_{2}+\omega_{3}+\omega_{4}, 0} \\
& \times\left\{-\frac{1}{4} y \bar{Y} I_{3,4}+2 \frac{g}{24} \bar{Y} I_{3,2}-\frac{g}{24} \bar{y} I_{3,2}+4 \frac{g^{2}}{24^{2}} I_{3,0}\right. \\
& +\left[-2 x^{2} y \bar{Y} I_{5,6}-8 \frac{g}{24} x^{2} \bar{y} I_{5,4}+16 \frac{g}{24} x^{2} \bar{Y} I_{5,4}+32 \frac{g^{2}}{24^{2}} x^{2} I_{5,2}\right. \\
& +\frac{5}{2} x y \bar{Y} I_{4,4}+6 \frac{g}{24} x \bar{y} I_{4,2}-12 \frac{g}{24} x \bar{Y} I_{4,2}-8 \frac{g^{2}}{24^{2}} x I_{4,0} \\
& \left.+\frac{1}{4} \bar{y}^{2} I_{3,2}-5 \frac{1}{4} \bar{y} \bar{Y} I_{3,2}+\frac{3}{2} \bar{Y}^{2} I_{3,2}+4 \frac{g}{24} \bar{Y} I_{3,0}-\frac{g}{24} \bar{y} I_{3,0}\right] \omega_{1}^{2} \\
& +\left[-2 x^{2} y \bar{Y} I_{5,6}-8 \frac{g}{24} x^{2} \bar{y} I_{5,4}+16 \frac{g}{24} x^{2} \bar{Y} I_{5,4}+32 \frac{g^{2}}{24^{2}} x^{2} I_{5,2}\right. \\
& +\frac{5}{2} x y \bar{Y} I_{4,4}+6 \frac{g}{24} x \bar{y} I_{4,2}-12 \frac{g}{24} x \bar{Y} I_{4,2}-8 \frac{g^{2}}{24^{2}} x I_{4,0} \\
& \left.\left.+\frac{1}{4} \bar{y}^{2} I_{3,2}-3 \frac{1}{4} \bar{y} \bar{Y} I_{3,2}+\bar{Y}^{2} I_{3,2}+2 \frac{g}{24} \bar{Y}_{\mu^{2}} I_{3,0}\right] \omega_{1} \omega_{2}\right\} \\
& \times q_{a, \omega_{1}} q_{a, \omega_{2}} q_{b, \omega_{3}} q_{b, \omega_{4}},
\end{aligned}
$$


and

$$
\begin{aligned}
T_{3}= & -8 \hbar \int_{\omega_{1}, \ldots, \omega_{4}, \omega} \delta_{\omega_{1}+\ldots+\omega_{4}, 0} \\
& \times\left[\frac{\bar{Y}^{2}}{4} I_{3,4}+\frac{g \bar{Y}}{12} I_{3,2}+4 \frac{g^{2}}{24^{2}} I_{3,0}\right. \\
& +\left(\bar{Y}^{2} x^{2} I_{5,6}+8 \frac{g \bar{Y}}{24} x^{2} I_{5,4}+16 \frac{g^{2}}{24^{2}} x^{2} I_{5,2}\right. \\
& -20 \frac{\bar{Y}^{2}}{16} x I_{4,4}-6 \frac{g \bar{Y}}{24} x I_{4,2}-4 \frac{g^{2}}{24^{2}} x I_{4,0} \\
& \left.+\bar{Y}^{2} I_{3,2}-\frac{1}{4} \bar{y}^{2} I_{3,2}-\frac{\bar{y} \bar{Y}}{4} I_{3,2}-\frac{g \bar{y}}{24} I_{3,0}+3 \frac{g \bar{Y}}{24} I_{3,0}\right) \omega_{1}^{2} \\
& +\left(-\bar{Y}^{2} x^{2} I_{5,6}-8 \frac{g \bar{Y}}{24} x^{2} I_{5,4}-16 \frac{g^{2}}{24^{2}} x^{2} I_{5,2}\right. \\
& +5 \frac{\bar{Y}^{2}}{4} x I_{4,4}+6 \frac{g \bar{Y}}{24} x I_{4,2}+4 \frac{g^{2}}{24^{2}} x I_{4,0} \\
& \left.\left.-\frac{3}{4} \bar{y}^{2} I_{3,2}-\frac{1}{2} \bar{Y}^{2} I_{3,2}+\frac{\bar{y} \bar{Y}}{4} I_{3,2}+\frac{g \bar{y}}{24} I_{3,0}-\frac{g \bar{Y}}{24} I_{3,0}\right) \omega_{1} \omega_{2}\right] \\
& \times q_{a, \omega_{1}} q_{a, \omega_{2}} q_{b, \omega_{3}} q_{b, \omega_{4}} .
\end{aligned}
$$

\section{Loop integrals}

In order to get the explicit forms of the RG equations (19)-(24) one has to perform the loop integrals (17),

$$
I_{n, s} \sim \int_{-\infty}^{\infty} \frac{d \omega}{2 \pi} G_{\omega}^{n} \omega^{s}=\left.\int_{-\infty}^{\infty} \frac{d \omega}{2 \pi x^{n}} \frac{\omega^{s}}{(\omega-a)^{n}(\omega+b)^{n}}\right|_{a=b=\sqrt{\left(\Omega^{2}-i \mu^{2}\right) / x}}
$$

For the limit $\Lambda \rightarrow \infty$ and for $s-2 n<-1$, like in our case, this can be done analytically. Since $\operatorname{Re} a>0, \operatorname{Im} a>0$, we can close the path of integration with a half-circle of infinite radius on the upper half of the complex $\omega$-plane encircling the multiple pole at $\omega=a$ and apply the residuum theorem,

$$
\frac{1}{2 \pi} \oint \frac{f(z)}{(z-a)^{n}}=\frac{i}{(n-1) !} \frac{d^{n-1}}{d a^{n-1}} f(a), \quad f(a)=\frac{a^{s}}{(a+b)^{n}} .
$$

Thus one finds

$$
I_{1,0}=\frac{i}{2 x^{\frac{1}{2}}\left(\Omega^{2}-i \mu^{2}\right)^{\frac{1}{2}}}, \quad I_{n, s}=\frac{i[s-(2 n-3)] \cdot(s-1)}{(n-1) ! 2^{n} x^{\frac{s+1}{2}}\left(\Omega-i \mu^{2}\right)^{n-\frac{s+1}{2}}} \quad \text { for } n>1
$$

which yield the relations

$$
\begin{aligned}
& I_{2,2}=\frac{1}{2 x} I_{1,0}, \quad I_{3,2}=\frac{1}{4 x} I_{2,0}, \quad I_{3,4}=\frac{3}{8 x^{2}} I_{1,0}, \quad I_{4,2}=\frac{1}{6 x} I_{3,0}, \quad I_{4,4}=\frac{1}{8 x^{2}} I_{2,0}, \\
& I_{5,2}=\frac{1}{8 x} I_{4,0}, \quad I_{5,4}=\frac{1}{16 x^{2}} I_{3,0}, \quad I_{5,6}=\frac{5}{64 x^{3}} I_{2,0}
\end{aligned}
$$

with

$$
I_{2,0}=\frac{-i}{4 x^{\frac{1}{2}}\left(\Omega-i \mu^{2}\right)^{\frac{3}{2}}}, \quad I_{3,0}=\frac{3 i}{16 x^{\frac{1}{2}}\left(\Omega-i \mu^{2}\right)^{\frac{5}{2}}}, \quad I_{4,0}=\frac{-5 i}{32 x^{\frac{1}{2}}\left(\Omega-i \mu^{2}\right)^{\frac{7}{2}}} .
$$

\section{Perturbation expansion}

Here we outline the solution of the evolution equations (25)-(29) for the $N=1$ dimensional oscillator in the second order of the perturbation expansion in the bare coupling $g_{B}$ after the analytic continuation $-i \mu^{2} \rightarrow \lambda$ to the Euclidean 
space. Making use of the result of the IMA, one can write the perturbation expansion of the couplings as,

$$
\begin{aligned}
\gamma & =\delta+g_{B} \alpha+g_{B}^{2} \beta+\ldots, \\
\delta \Omega^{2} & =g_{B} \rho+g_{B}^{2} \sigma+\ldots, \\
x-1 & =g_{B}^{2} \zeta+\ldots, \\
\Upsilon & =g_{B}^{2} \eta, \\
g & =g_{B}+g_{B}^{2} \nu
\end{aligned}
$$

with $\delta \Omega^{2}=\Omega^{2}-\omega_{0}^{2}$, and using $x-1 \sim \mathcal{O}(\Upsilon) \sim \mathcal{O}\left(g_{B}^{2}\right)$. For the sake of simplicity, we shall set $\hbar=1$ in this section. In order to make explicit the dependence on the bare coupling $g_{B}$ in the right-hand sides of Eqs. (25)-(29), one has to expand the denominators of the loop integrals given by Eq. (A14),

$$
\begin{aligned}
\frac{1}{\left(\Omega^{2}+\lambda\right)^{a}} & =\frac{1}{\left(\omega_{0}^{2}+\lambda\right)^{a}}-a \frac{g_{B} \rho}{\left(\omega_{0}^{2}+\lambda\right)^{a+1}}+g_{B}^{2}\left(\frac{1}{2} a(a+1) \frac{\rho^{2}}{\left(\omega_{0}^{2}+\lambda\right)^{a+2}}-\frac{a \sigma}{\left(\omega_{0}^{2}+\lambda\right)^{a+1}}\right), \\
x^{a} & \approx 1+a g_{B}^{2} \zeta+\ldots,
\end{aligned}
$$

where the relation

$$
\frac{1}{(1+x)^{a}} \approx 1-a x+\frac{1}{2} a(a+1) x^{2}+\ldots
$$

has been used. The comparison of the coefficients of the corresponding powers of $g_{B}$ in both sides of the Eqs. (25)-(29) yields the following set of coupled ordinary first-order differential equations for the running couplings,

$$
\begin{gathered}
\partial_{\lambda} \delta=\frac{1}{4\left(\omega_{0}^{2}+\lambda\right)^{\frac{1}{2}}}, \\
\partial_{\lambda} \alpha=-\frac{1}{8} \frac{\rho}{\left(\omega_{0}^{2}+\lambda\right)^{\frac{3}{2}}}, \\
\partial_{\lambda} \beta=\frac{3}{32} \frac{\rho^{2}}{\left(\omega_{0}^{2}+\lambda\right)^{\frac{5}{2}}}+\frac{1}{8} \frac{\sigma}{\left(\omega_{0}^{2}+\lambda\right)^{\frac{3}{2}}}+\frac{1}{8} \frac{\zeta}{\left(\omega_{0}^{2}+\lambda\right)^{\frac{1}{2}}}, \\
\partial_{\lambda} \zeta=-\frac{\eta}{4\left(\omega_{0}^{2}+\lambda\right)^{\frac{3}{2}}}, \\
\partial_{\lambda} \eta=-\frac{5}{64\left(\omega_{0}^{2}+\lambda\right)^{\frac{7}{2}}}, \\
\partial_{\lambda} \rho=-\frac{1}{8\left(\omega_{0}^{2}+\lambda\right)^{\frac{3}{2}}}, \\
\partial_{\lambda} \sigma=-\frac{\nu}{4\left(\omega_{0}^{2}+\lambda\right)^{\frac{1}{2}}}-\frac{\nu}{8\left(\omega_{0}^{2}+\lambda\right)^{\frac{3}{2}}}+\frac{3}{16} \frac{\rho}{\left(\omega_{0}^{2}+\lambda\right)^{\frac{5}{2}}}, \\
\frac{9}{16\left(\omega_{0}^{2}+\lambda\right)^{\frac{5}{2}}} .
\end{gathered}
$$


The solutions belonging to the initial conditions $\delta_{B}=-\frac{1}{2}\left(\omega_{0}^{2}+\lambda_{B}\right)^{\frac{1}{2}}, \alpha_{B}=\beta_{B}=\zeta_{B}=\eta_{B}=\rho_{B}=\sigma_{B}=\nu_{B}=0$ at $\lambda_{B} \rightarrow \infty$ and their IR limits $\lambda \rightarrow 0$ have been found analytically,

$$
\begin{aligned}
& \delta=\frac{1}{2}\left(\omega_{0}^{2}+\lambda\right)^{\frac{1}{2}} \rightarrow \frac{1}{2} \omega_{0}, \\
& \eta=\frac{1}{32\left(\omega_{0}^{2}+\lambda\right)^{\frac{5}{2}}} \rightarrow \frac{1}{32 \omega_{0}^{5}}, \\
& \rho=\frac{1}{4\left(\omega_{0}^{2}+\lambda\right)^{\frac{1}{2}}} \rightarrow \frac{1}{4 \omega_{0}}, \\
& \nu=-\frac{3}{8\left(\omega_{0}^{2}+\lambda\right)^{\frac{3}{2}}} \rightarrow-\frac{3}{8 \omega_{0}^{3}}, \\
& \sigma=-\frac{11}{256\left(\omega_{0}^{2}+\lambda\right)^{2}} \rightarrow-\frac{11}{256 \omega_{0}^{4}}, \\
& \alpha=\frac{1}{32\left(\omega_{0}^{2}+\lambda\right)} \rightarrow \frac{1}{32 \omega_{0}^{2}}, \\
& \zeta=\frac{1}{3 \cdot 128\left(\omega_{0}^{2}+\lambda\right)^{3}} \rightarrow \frac{1}{3 \cdot 128 \omega_{0}^{6}}, \\
& \beta=-\frac{71}{3 \cdot 5 \cdot 1024\left(\omega_{0}^{2}+\lambda\right)^{\frac{5}{2}}} \rightarrow-\frac{71}{3 \cdot 5 \cdot 1024 \omega_{0}^{5}} .
\end{aligned}
$$

Then one obtains for the energy of the ground state

$$
E_{0}=\gamma_{0}=\frac{1}{2} \omega_{0}\left[1+\xi-\frac{71}{30} \xi^{2}\right]
$$

for the frequency parameter

$$
\Omega_{0}^{2}=\omega_{0}^{2}\left(1+4 \xi-11 \xi^{2}\right)
$$

the renormalized quartic coupling

$$
g_{0}=g_{B}(1-6 \xi),
$$

and the couplings for the wavefunction renormalization

$$
x_{0}=1+\frac{2}{3} \xi^{2}, \quad \hbar \Upsilon_{0}=8 \omega_{0} \xi^{2}
$$

where we reestablished the powers of $\hbar$.

\section{Localization}

The localization of the ground state wavefunction can be characterized by its momenta. We can make an estimate of the dependence of the variance of the coordinate operator on the dimension $N$ of the oscillator. Let us write for it with the help of the Lehmann-expansion of the propagator,

$$
\begin{aligned}
\left\langle 0\left|\underline{q}^{2}\right| 0\right\rangle & =\sum_{a=1}^{N}\left\langle 0\left|q_{a}^{2}\right| 0\right\rangle=\lim _{t \rightarrow 0^{+}} \sum_{a=1}^{N} \sum_{n, \alpha}\left\langle 0\left|q_{a}(t)\right| n, \alpha\right\rangle\left\langle 0, \alpha\left|q_{a}(0)\right| 0\right\rangle \\
& =\lim _{t \rightarrow 0^{+}} \sum_{a=1}^{N} \sum_{n, \alpha} e^{i\left(E_{0}-E_{n}\right) t / \hbar}\left|\left\langle 0\left|q_{a}\right| n, \alpha\right\rangle\right|^{2} \\
& =\sum_{a=1}^{N} \sum_{n, \alpha} \lim _{t \rightarrow 0^{+}} \int_{\omega} e^{i \omega t} \frac{2\left(E_{n}-E_{0}\right) / \hbar}{\omega^{2}+\left[\left(E_{n}-E_{0}\right) / \hbar\right]^{2}}\left|\left\langle 0\left|q_{a}\right| n, \alpha\right\rangle\right|^{2}
\end{aligned}
$$

where $H|n, \alpha\rangle=E_{n}|n, \alpha\rangle$ for the exact eigenstates $|n, \alpha\rangle$ and energy levels $E_{n}$ of the Hamiltonian, $|0\rangle$ and $E_{0}$ stand for the ground state and its energy, $\alpha$ are additional quantum numbers for counting states belonging to the same 
degenerate energy level. Operators without and with the time argument stand for those in the Schrödinger and the Heisenberg representations, respectively. Making use of the dominance of the pole of the integrand corresponding to the first excited state [27], one finds the estimate

$$
\begin{aligned}
\left\langle 0\left|\underline{q}^{2}\right| 0\right\rangle & \approx \lim _{t \rightarrow 0^{+}} \int_{\omega} \frac{e^{i \omega t}}{\omega^{2}+\left(\Omega^{2} / x\right)} \sum_{a=1}^{N} \frac{1}{\hbar} \sum_{n, \alpha} 2\left(E_{n}-E_{0}\right)\left|\left\langle 0\left|q_{a}\right| n, \alpha\right\rangle\right|^{2} \\
& =\frac{x^{\frac{1}{2}}}{2 \Omega} \sum_{a=1}^{N} \frac{1}{\hbar} \sum_{n, \alpha} 2\left(E_{n}-E_{0}\right)\left|\left\langle 0\left|q_{a}\right| n, \alpha\right\rangle\right|^{2} .
\end{aligned}
$$

Making use of the Thomas-Reiche-Kuhn sum rule [40], valid for any self-adjoint operator $A$,

$$
\frac{1}{2}\langle 0|[[M, H], M]| 0\rangle=\sum_{n, \alpha}\left(E_{n}-E_{0}\right)|\langle 0|M| n, \alpha\rangle|^{2}
$$

and the form of the renormalized Hamilton-operator $H=\frac{1}{2} x p^{2}+V(q)$ where we neglected the less important highermomentum terms in respect to the small values of $\bar{y}$ and $\bar{Y}$, we get

$$
\sum_{n, \alpha}\left(E_{n}-E_{0}\right)\left|\left\langle 0\left|q_{a}\right| n, \alpha\right\rangle\right|^{2}=\frac{1}{2}\left\langle 0\left|\left[\left[q_{a}, H\right], q_{a}\right]\right| 0\right\rangle=\frac{x}{4}\left\langle 0\left|\left[\left[q_{a}, p_{a}^{2}\right], q_{a}\right]\right| 0\right\rangle=\frac{1}{2} x \hbar^{2}
$$

where there is no summation over the index $a$. With the help of this relation we obtain

$$
\left\langle 0\left|\underline{q}^{2}\right| 0\right\rangle \approx \frac{x^{\frac{1}{2}}}{2 \Omega} \frac{2}{\hbar} \sum_{a=1}^{N} \frac{1}{2} x \hbar^{2}=\frac{N x^{3 / 2} \hbar}{2 \Omega} .
$$

\section{Appendix B: WH RG flow of the Wilsonian action in Euclidean space}

\section{Blocking via mode-by-mode integration}

The WH RG method [29] has been applied to simple quantum mechanical systems with success in the literature [27]. The simplicity of the $0+1$ dimensional model of the single quantized anharmonic oscillator enables one to perform the blocking by integrating out the high-frequency quantum fluctuations mode-by-mode. Let us prescribe periodic boundary conditions in time, $q_{t=0}=q_{t=T}, \xi_{t=0}=\xi_{t=T}$ both for the coordinate $q_{t}$ and the mode $\xi_{t}$ integrated out in a single blocking step, where $T$ stand for the time interval the action is integrated over and taken to infinity at the end. The Matsubara frequencies are given as $\omega_{n}=n 2 \pi / T$, the gliding cut-off is at $\omega_{N}=N 2 \pi / T$. The periodic coordinate can be Fourier expanded as

$$
q_{t}=\frac{a_{0}}{2}+\sum_{n=1}^{N_{\Lambda}} a_{n} \cos \left(\omega_{n} t\right)+\sum_{n=1}^{N_{\Lambda}} b_{n} \sin \left(\omega_{n} t\right)=\bar{q}+\eta_{t}
$$

where $\omega_{N_{\Lambda}}=N_{\Lambda} 2 \pi / T=\Lambda$ is the UV cut-off. Integrating out all the quantum fluctuations $\eta_{t}$ around the constant mode $\bar{q}=\frac{1}{2} a_{0}$ leads to the effective action $S_{0}(\bar{q})$ given by

$$
\begin{aligned}
e^{-\frac{1}{\hbar} S_{0}(\bar{q})} & =\int \mathcal{D} \eta e^{-\frac{1}{\hbar} S_{\Lambda}[\bar{q}+\eta]} \\
& \sim\left(\prod_{n=1}^{N_{\Lambda}} \int_{-\infty}^{\infty} d a_{n} \int_{-\infty}^{\infty} d b_{n}\right) e^{-\frac{1}{\hbar} S_{\Lambda}[\bar{q}+\eta]}
\end{aligned}
$$

up to an irrelevant constant being independent of $\bar{q}$ and the potential (see [42]). The blocking relation for integrating out the single mode with frequency $\omega_{N}$, ie. the mode $\xi_{t}=a_{N} \cos \left(\omega_{N} t\right)+b_{N} \sin \left(\omega_{N} t\right)$ is given by

$$
e^{-\frac{1}{\hbar} S_{N-1}[\bar{q}]}=\int \mathcal{D} \xi e^{-\frac{1}{\hbar} S_{N}[\bar{q}+\xi]} \sim \int_{-\infty}^{\infty} d a_{N} \int_{-\infty}^{\infty} d b_{N} e^{-\frac{1}{\hbar} S_{N}[\bar{q}+\xi]}
$$


in the LPA. Here the LPA enables one to replace the time-dependent background by the constant mode $\bar{q}$. The kinetic term of the action gives

$$
\int_{0}^{T} d t \frac{1}{2}\left(\dot{q}_{0}+\dot{\xi}_{t}\right)^{2}=\frac{T}{4} \omega_{N}^{2}\left[a_{N}^{2}+b_{N}^{2}\right]
$$

With the notation $a_{\sigma=1}=a_{N}, a_{\sigma=-1}=b_{N}$, we can write for the blocked potential

$$
\mathcal{U}_{N}[\bar{q}+\xi]=\int_{t} U_{N}\left(\bar{q}+\xi_{t}\right)=T U_{N}(\bar{q})+\frac{1}{2} \sum_{\sigma, \sigma^{\prime}= \pm 1} a_{\sigma} a_{\sigma^{\prime}} \mathcal{U}_{N \sigma, \sigma^{\prime}}^{(2)}(\bar{q})+\mathcal{O}\left(\xi^{3}\right)
$$

where

$$
\mathcal{U}_{N \sigma, \sigma^{\prime}}^{(2)}(\bar{q})=\left.\frac{\delta^{2}}{\delta a_{\sigma}^{\prime} \delta a_{\sigma}} \int_{t} U_{N}\left(\bar{q}+\xi_{t}\right)\right|_{\xi=0}=T \frac{1}{2} U_{N}^{(2)}(\bar{q})\left[\delta_{\sigma, 1} \delta_{\sigma^{\prime}, 1}+\delta_{\sigma,-1} \delta_{\sigma^{\prime},-1}\right]
$$

so that one gets

$$
\mathcal{U}_{N}[\bar{q}+\xi]=T U_{N}(\bar{q})+T \frac{1}{4} U_{N}^{(2)}(\bar{q})\left[a_{N}^{2}+b_{N}^{2}\right]+\mathcal{O}\left(\xi^{3}\right)
$$

The non-Gaussian higher-order terms are suppressed with some powers of $1 / T$ in the path-integral and can be neglected. Then the Gaussian path integral can be performed,

$$
\begin{aligned}
e^{-\frac{1}{\hbar} T U_{N-1}(\bar{q})} & \sim e^{-\frac{1}{\hbar} T U_{N}(\bar{q})} \int_{-\infty}^{\infty} d a_{N} \int_{-\infty}^{\infty} d b_{N} e^{-\frac{1}{\hbar} T \frac{1}{4}\left[\omega_{N}^{2}+U_{N}^{(2)}(\bar{q})\right]\left[a_{N}^{2}+b_{N}^{2}\right]} \\
& \sim e^{-\frac{1}{\hbar} T U_{N}(\bar{q})}\left(\sqrt{\frac{8 \pi \hbar}{T\left[\omega_{N}^{2}+U_{N}^{(2)}(\bar{q})\right]}}\right)^{2}
\end{aligned}
$$

yielding the finite difference equation

$$
\frac{U_{N}(\bar{q})-U_{N-1}(\bar{q})}{2 \pi / T}=\frac{U_{\omega_{N}}\left(\bar{q}-U_{\omega_{N}-\Delta \omega}(\bar{q})\right.}{\Delta \omega}=-\frac{\hbar / T}{2 \pi / T} \ln \left[\omega_{N}^{2}+U_{N}^{(2)}(\bar{q})\right]
$$

for the blocked potential at the scale $\omega_{N}$. In the limit $T \rightarrow \infty$, ie. $\Delta \omega \rightarrow 0$ one finds the WH RG equation

$$
\partial_{\omega} U_{\omega}(\bar{q})=-\hbar \alpha \ln \left[\omega^{2}+U_{\omega}^{(2)}(\bar{q})\right], \quad \alpha=\frac{1}{2 \pi}
$$

for the blocked potential $U_{\omega}$, where the discrete gliding frequency cut-off $\omega_{N}$ has been changed to the continuous one, denoted by $\omega$.

\section{Approximation schemes}

$$
\text { a. IMA }
$$

In the IMA one has to replace the blocked potential in the right-hand side by the bare one. Then one can perform the integration over the scale $\omega$ in a straightforward manner,

$$
U_{\omega}^{\mathrm{IMA}}(\bar{q})=U_{\Lambda}(\bar{q})+\hbar \alpha \int_{\omega}^{\Lambda} d k \ln \left[k^{2}+a^{2}\right], \quad a^{2}=U_{\Lambda}^{(2)}(\bar{q}) .
$$

The bare potential is convex at least in the neighbourhood of its minimum (minima) at some $q_{0}$. Then for $\bar{q}$ sufficiently close to $q_{0}$, one can make use of the integral

$$
\int_{\omega}^{\Lambda} d k \ln \left[k^{2}+a^{2}\right]=\left[k \ln \left[k^{2}+a^{2}\right]-2 k+2 a \arctan \frac{k}{a}\right]_{\omega}^{\Lambda} \stackrel{\Lambda \rightarrow \infty, \omega \rightarrow 0}{\longrightarrow} 2 \Lambda(\ln \Lambda-1)+a \pi
$$


and find for the effective potential

$$
U_{0}^{\mathrm{IMA}}(\bar{q})=U_{\Lambda}(\bar{q})+\hbar \pi \sqrt{U_{\Lambda}^{(2)}(\bar{q})} .
$$

This expression is valid in the neighbourhood of the minimum $q_{0}$ of the bare potential, and the UV divergent piece is removed by the renormalization condition that the effective potential of a free particle $\left(U_{\Lambda}(\bar{q})=0, a=0\right)$ were identically vanishing.

The minimum at $q_{0}$ of the bare potential would be displaced to $q_{0}^{I M A}=q_{0}+\delta q$ with

$$
\delta q=-\frac{\hbar \pi U_{\Lambda}^{(3)}\left(q_{0}\right)}{2\left[U_{\Lambda}^{(2)}\left(q_{0}\right)\right]^{3 / 2}}
$$

so that for a potential with the symmetry $\bar{q} \rightarrow-\bar{q}$ and its minimum at $q_{0}=0$ the minimum is not displaced in the one-loop order. Then one finds for the ground state energy

$$
E_{0}^{\mathrm{IMA}}=\hbar \alpha \pi \sqrt{U_{\Lambda}^{(2)}(0)}
$$

in the IMA, ie. in the one-loop approximation. For the free motion of a particle the bare potential vanishes and it remains vanishing after the blocking as well. For the case of a linear harmonic oscillator this reproduces the correct ground state energy $E_{0}^{\mathrm{IMA} \text {, lho. }}=\frac{1}{2} \hbar \omega_{0}$. For an arbitrary polynomial bare potential $U_{\Lambda}(\bar{q})=\frac{1}{2} \omega_{0}^{2} \bar{q}^{2}+\frac{1}{24} g_{B} \bar{q}^{4}+\ldots$ we get

$$
U_{0}^{\mathrm{IMA}, \text { aho. }}(\bar{q})=U_{\Lambda}(\bar{q})+\frac{1}{2} \hbar \omega_{0}\left(1+\frac{g_{B} \bar{q}^{2}}{4 \omega_{0}^{2}}+\ldots\right)=\frac{1}{2} \hbar \omega_{0}+\frac{1}{2}\left(\omega_{0}^{2}+\frac{g_{B} \hbar}{4 \omega_{0}}\right) \bar{q}^{2}+\frac{1}{24} g_{B} \bar{q}^{4}+\ldots
$$

This means that the energy of the ground state of the anharmonic oscillator remains the same as that of the harmonic oscillator, but the excitation energy of the first excited state is increased with increasing coupling $g_{B}$,

$$
\Delta E^{\mathrm{IMA}}=\hbar \sqrt{\omega_{0}^{2}+\frac{g_{B} \hbar}{4 \omega_{0}}} \approx \hbar \omega_{0}\left(1+\frac{g_{B} \hbar}{8 \omega_{0}^{3}}+\mathcal{O}\left(g_{B}^{2}\right)\right)
$$

in the IMA. The same result has been obtained in the CSi RG scheme in the IMA.

\section{b. Perturbation expansion in powers of the bare coupling $g_{B}$}

Let us introduce the blocked interaction potential $V_{\omega}(\bar{q})$ via

$$
U_{\omega}(\bar{q})=u_{\omega}+\frac{1}{2} \omega_{0}^{2} \bar{q}^{2}+V_{\omega}(\bar{q})
$$

for which the WH-RG equation (B10) can be rewritten as

$$
\partial_{\omega} u_{\omega}+\partial_{\omega} V_{\omega}(\bar{q})=-\hbar \alpha \ln \left[\omega^{2}+\omega_{0}^{2}+V_{\omega}^{\prime \prime}(\bar{q})\right]=-\hbar \alpha \ln \left(\omega^{2}+\omega_{0}^{2}\right)-\hbar \alpha \sum_{n=1}^{\infty} \frac{-(-1)^{n}}{n}\left[G_{0}{ }_{\omega} V_{\omega}^{\prime \prime}(\bar{q})\right]^{n} .
$$

In the last equation we performed the perturbation expansion of the right hand side into the interaction, $G_{0}$ denotes the propagator of the harmonic oscillator in the ground state. Further on let us Taylor-expand the blocked interaction potential into the powers of $\bar{q}$ and truncate it at the quartic terms,

$$
V_{\omega}(\bar{q})=g_{B} \alpha_{\omega}+g_{B}^{2} \beta_{\omega}+\frac{1}{2}\left(g_{B} \rho_{\omega}+g_{B}^{2} \sigma_{\omega}\right) \bar{q}^{2}+\frac{1}{24}\left(g_{B}+g_{B}^{2} \nu_{\omega}\right) \bar{q}^{4}+\ldots,
$$

and Taylor-expand each running coupling into the powers of the bare coupling $g_{B}$ and truncate their perturbation serii at the second order terms. Inserting this ansatz into Eq. (B19) and keeping all the terms up to the order $\bar{q}^{4}$ and $g_{B}^{2}$, one finds the following set of coupled ordinary differential equations for the RG flow of the various couplings 
$(\cdot=d / d \omega)$,

$$
\begin{aligned}
& \dot{u}_{\omega}=-\hbar \alpha \ln \left(\omega^{2}+\omega_{0}^{2}\right) \\
& \dot{\alpha}_{\omega}=-\hbar \alpha \frac{\rho_{\omega}}{\omega^{2}+\omega_{0}^{2}} \\
& \dot{\beta}_{\omega}=-\hbar \alpha \frac{\sigma_{\omega}}{\omega^{2}+\omega_{0}^{2}}+\frac{1}{2} \hbar \alpha \frac{\rho_{\omega}^{2}}{\left(\omega^{2}+\omega_{0}^{2}\right)^{2}}, \\
& \dot{\rho}_{\omega}=-\hbar \alpha \frac{1}{\omega^{2}+\omega_{0}^{2}}, \\
& \dot{\sigma}_{\omega}=-\hbar \alpha \frac{\nu_{\omega}}{\omega^{2}+\omega_{0}^{2}}+\hbar \alpha \frac{\rho_{\omega}}{\left(\omega^{2}+\omega_{0}^{2}\right)^{2}} \\
& \dot{\nu}_{\omega}=3 \hbar \alpha \frac{1}{\left(\omega^{2}+\omega_{0}^{2}\right)^{2}}
\end{aligned}
$$

with the initial conditions $\alpha_{B}=\beta_{B}=\rho_{B}=\sigma_{B}=\nu_{B}=0$ at the UV cut-off $\omega=\Lambda$. Here the equations for $u_{\omega}, \rho_{\omega}, \nu_{\omega}, \alpha_{\omega}$ can be integrated straightforwardly,

$$
\begin{gathered}
u_{\omega}=u_{\Lambda}+\hbar \alpha \int_{\omega}^{\Lambda} d k \ln \left(k^{2}+\omega_{0}^{2}\right) \stackrel{\Lambda \rightarrow \infty, \omega \rightarrow 0}{\longrightarrow} \mathrm{UV} \text { div. terms }+\frac{1}{2} \hbar \omega_{0}, \\
\rho_{\omega}=\frac{\hbar}{2 \pi} \int_{\omega}^{\Lambda} \frac{d k}{k^{2}+\omega_{0}^{2}}=\frac{\hbar}{2 \pi \omega_{0}}\left[\arctan \frac{k}{\omega_{0}}\right]_{\omega}^{\Lambda} \stackrel{\Lambda \rightarrow \infty, \omega \rightarrow 0}{\longrightarrow} \frac{\hbar}{4 \omega_{0}}, \\
\nu_{\omega}=-\frac{3 \hbar}{2 \pi} \int_{\omega}^{\Lambda} \frac{d k}{\left(k^{2}+\omega_{0}^{2}\right)^{2}}=-\frac{3 \hbar}{2 \pi}\left[\frac{k}{2 \omega_{0}^{2}\left(k^{2}+\omega_{0}^{2}\right)}+\frac{1}{2 \omega_{0}^{3}} \arctan \frac{k}{\omega_{0}}\right]_{\omega}^{\Lambda} \stackrel{\Lambda \rightarrow \infty, \omega \rightarrow 0}{\longrightarrow}-\frac{3 \hbar}{8 \omega_{0}^{3}}, \\
\alpha_{\omega}=\frac{\hbar^{2} \alpha^{2}}{\omega_{0}} \int_{\omega}^{\Lambda} d k \frac{\arctan \frac{\Lambda}{\omega_{0}}-\arctan \frac{k}{k_{0}}}{k^{2}+\omega_{0}^{2}} \\
\left.=\frac{\hbar^{2} \alpha^{2}}{\omega_{0}}\left[\frac{1}{\omega_{0}} \arctan \frac{\Lambda}{\omega_{0}}\left(\arctan \frac{k}{\omega_{0}}\right)_{\omega}^{\Lambda}-\frac{1}{2 \omega_{0}} \int_{\omega}^{\Lambda} d k 2\left(\arctan \frac{k}{\omega_{0}}\right)^{\prime} \arctan \frac{\Lambda}{\omega_{0}}\left(\arctan \frac{k}{\omega_{0}}\right)_{\omega}^{\Lambda}-\frac{1}{2 \omega_{0}}\left(\arctan \frac{k}{\omega_{0}}\right)_{\omega}^{\Lambda}\right] \stackrel{\hbar^{2}}{\longrightarrow}\right]
\end{gathered}
$$

The rest of the equations cannot be integrated straightforwardly, but their solutions can be approximated by inserting the IR limits of the couplings $\rho_{\omega}, \nu_{\omega}$ into their right hand sides,

$$
\begin{gathered}
\sigma_{\omega} \approx-\frac{3 \hbar^{2}}{16 \pi \omega_{0}^{3}} \int_{\omega}^{\Lambda} \frac{d k}{k^{2}+\omega_{0}^{2}}-\frac{\hbar^{2}}{8 \pi \omega_{0}} \int_{\omega}^{\Lambda} \frac{d k}{\left(k^{2}+\omega_{0}^{2}\right)^{2}} \stackrel{\Lambda \rightarrow \infty, k \rightarrow 0}{\longrightarrow}-\frac{\hbar^{2}}{8 \omega_{0}^{4}}, \\
\beta_{\omega} \approx \frac{\hbar}{2 \pi} \frac{-\hbar^{2}}{8 \omega_{0}^{4}} \int_{\omega}^{\Lambda} \frac{d k}{k^{2}+\omega_{0}^{2}}-\frac{\hbar}{4 \pi} \frac{\hbar^{2}}{16 \omega_{0}^{2}} \int_{\omega}^{\Lambda} \frac{d k}{\left(k^{2}+\omega_{0}^{2}\right)^{2}} \stackrel{\Lambda \rightarrow \infty, k \rightarrow 0}{\longrightarrow}-\frac{9 \hbar^{3}}{256 \omega_{0}^{5}} .
\end{gathered}
$$

Finally one finds

$$
\begin{aligned}
E_{0} & =u_{0}+g_{B} \alpha_{0}+g_{B}^{2} \beta_{0}=\frac{1}{2} \hbar \omega_{0}\left(1+\xi-18 \xi^{2}+\ldots\right), \\
\Omega_{0}^{2} & =\omega_{0}^{2}+g_{B} \rho_{0}+g_{B}^{2} \sigma_{0}=\omega_{0}^{2}\left(1+4 \xi-32 \xi^{2}+\ldots\right), \\
g_{0} & =g_{B}+g_{B}^{2} \nu_{0}=g_{B}(1-6 \xi+\ldots)
\end{aligned}
$$

in terms of the dimensionless parameter $\xi=g_{B} \hbar /\left(16 \omega_{0}^{3}\right)$. One should mention that the gradient expansion cannot be improved going beyond the LPA in the framework of the WH RG scheme. 